\title{
Turonian Radiolarians from Karnezeika, Argolis Peninsula, Peloponnesus (Greece)
}

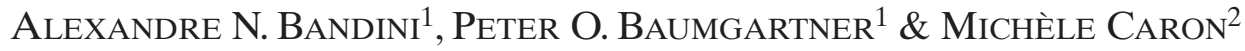

Key words: Mesozoic, Upper Cretaceous, Turonian, Radiolaria, Foraminifera, Tethys, Pelagonian Zone, Argolis Peninsula, Greece

\begin{abstract}
Near Karnezeika a roughly $140 \mathrm{~m}$ thick Upper Cretaceous section consists of interbedded pelagic limestones, cherts and coarse polymict breccias including ophiolites and shallow water limestones. At the base, pink pelagic limestones rest on deeply altered and fractured Lower Jurassic Pantokrator Limestone. This first pelagic facies is dated as middle Turonian, based on planktonic Foraminifera. Over $100 \mathrm{~m}$ of coarse ophiolite-carbonate breccias, interpreted as a channel or canyon fill in a pelagic environment, document the erosion of the Late Jurassic nappe edifice along the Cretaceous Pelagonian margin. Above these breccias, we mesured $16 \mathrm{~m}$ of principally pink and red pelagic limestones and radiolarian cherts, in which we recovered well-preserved radiolarians discussed here. In this interval, the presence of planktonic Foraminfera allows to state a late Turonian to Coniacian age. More than 40 radiolarian
\end{abstract}

species are described and figured in this work. The radiolarian chronostratigraphy established by 10 different authors in 11 publications was compared for this study and used to establish radiolarian ranges. This exercise shows major discrepancies between authors for the radiolarian ranges of the studied assemblage. Nevertheless, a Turonian age can be stated based on a synthesis of cited radiolarian ranges. This age is consistent with the age based on planktonic foraminifera. In combining the ages of both Radiolaria and planktonic Foraminifera, the studied samples can be restricted to the late Turonian. However, the discrepancies of published radiolarian ranges call for an urgent, major revision of the Late Cretaceous radiolarian biochronology. The integration of planktonic foraminifera with radiolarians may greatly enhance biochronologic resolution in sections where both groups occur.

\section{Introduction}

Relatively few studies exist of Upper Cretaceous Radiolaria from Greece, except for some occurences in the PindosOlonos zone (De Wever \& Thiébault 1981; Thiébault et al. 1981; De Wever \& Origlia-Devos 1982; Neumann 2003). In the Hellenides, ongoing tectonic activity is reflected in a smallscaled puzzle of shallow, detrital and pelagic facies in palaeogeographic realms such as the Pelagonian (Vrielynck 1981). Upper Cretaceous radiolarian occurrences are therefore restricted to times of high silica producticity in pelagic palaeoenvironments. One of the objectives of this study was to compare published radiolarian ranges given by different authors for the Upper Cretaceous and to try to establish an acceptable radiolarian age in spite of the differences in radiolarian ranges given by the various authors.

For this preliminary work we collected 17 samples in a total of $140 \mathrm{~m}$ of section for the study of planktonic Foraminifera and Radiolaria. 5 samples in a $2.6 \mathrm{~m}$ interval above coarse ophiolite-carbonate breccias yielded well-preserved radiolarians. From these samples 41 radiolarian species belonging to 18 genera are described and figured in this work. Overall, the assemblage resembles those described by O'Dogherty (1994) from the lower Turonian. The radiolarian biostratigraphy established by the following 11 publications (given with regions) was compared for this study: Dumitrica (1975, Romania), Foreman (1975, Pacific and 1977, Atlantic), O'Dogherty (1994, Italy and Spain), Pessagno (1976, California), Riedel \& Sanfilippo (1974, Composite), Sanfilippo \& Riedel (1985, Composite), Schaaf (1985, Composite), Taketani (1982, Japan), Thurow (1988, Atlantic), Vishnevskaya (2001, Russia).

The presence of abundant planktonic Foraminifera both at the base of the studied Cretaceous section and immediately above the radiolarian samples allow the comparison between the ages determined by radiolarian and foraminiferal biostratigraphy (Caron 1985).

\footnotetext{
${ }^{1}$ Institut de géologie et de paléontologie, Anthropole-Dorigny, Université de Lausanne, 1015 Lausanne, Switzerland. Email: AlexandreNicolas.Bondini@unil.ch,Peter.Baumgartner@unil.ch

${ }^{2}$ Institut de géologie et de paléotonlogie, Université de Fribourg, Pérolles, 1700 Fribourg, Switzerland. Email: Michele.Caron@unifr.ch
} 


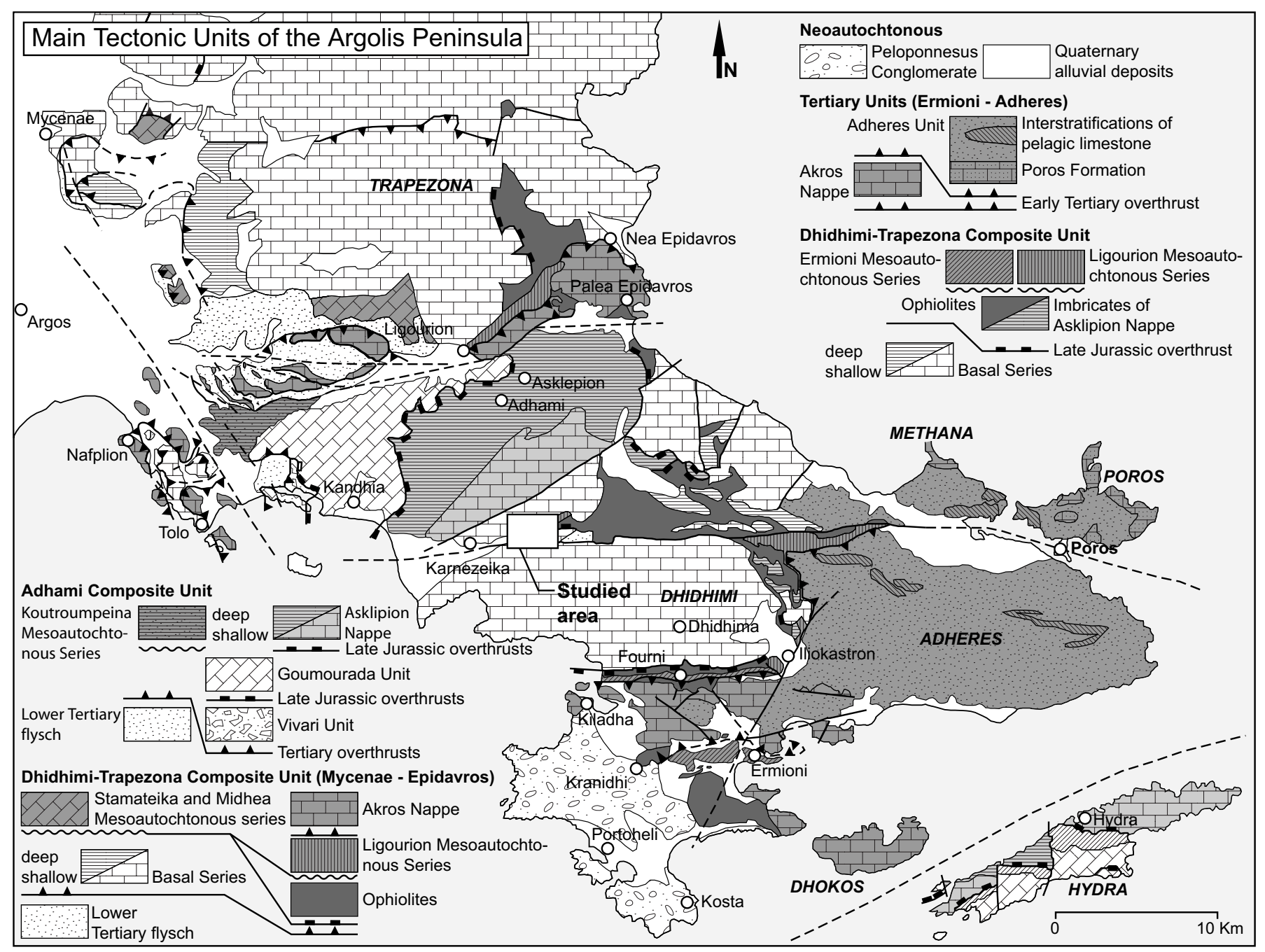

Fig. 1. Overview of the tectono-stratigraphy of the Argolis Peninsula. Based on: Baumgartner (1985), Vrielynck (1981), Clift (Poros Island) and several unpublished diploma thesis University of Lausanne (Bandini 2004; Giraud 2005; Glassey 2005).

\section{Geological Setting}

In the Argolis Peninsula, the Jurassic nappe edifice of the eastern Pelagonian margin is unconformably overlain by several different Cretaceous series (Vrielynck 1981). The studied section is located in the Depression of Karnezeika-Stavropodhi, a complex nappe syncline affected by neotectonic E-W trending subvertical faults (Fig. 1). The northern edge of this zone is built by Upper Triassic to Lower Jurassic Pantokrator Limestone (Fig. 2) belonging to the Basal Series of the DhidhimiTrapezona Composite Unit (Baumgartner 1985). These series became overthrust during the late Jurassic by nappes including ophiolites and then the area became deeply eroded during latest Jurassic and Early Cretaceous times.

Upper Cretaceous pelagic and coarse clastic sediments unconformably overlie the Pantokrator limestone of the basal series and contain disorganised boulder breccias of basalts and shallow water limestones that document the ongoing erosion of the Late Jurassic Pelagonian nappe edifice in a high-relief, deeper marine environment.

\section{Stratigraphy and sedimentology}

Near Karnezeika a roughly $140 \mathrm{~m}$ thick section consists of interbedded pelagic limestones, cherts and boulder breccias containing abundant ophiolite clasts along with boulders of the underlying Pantokrator Limestone (Fig. 3). At the base, pink pelagic limestones rest on deeply altered and fractured Pantokrator Limestone. Over $100 \mathrm{~m}$ of coarse ophiolite-carbonate breccias represent a channel or canyon fill in a pelagic environment. The following $16 \mathrm{~m}$ are principally pink and red pelagic limestones and radiolarian cherts, from which we recovered well-preserved radiolarians described here.

The studied section rests conformably on the breccias de- 


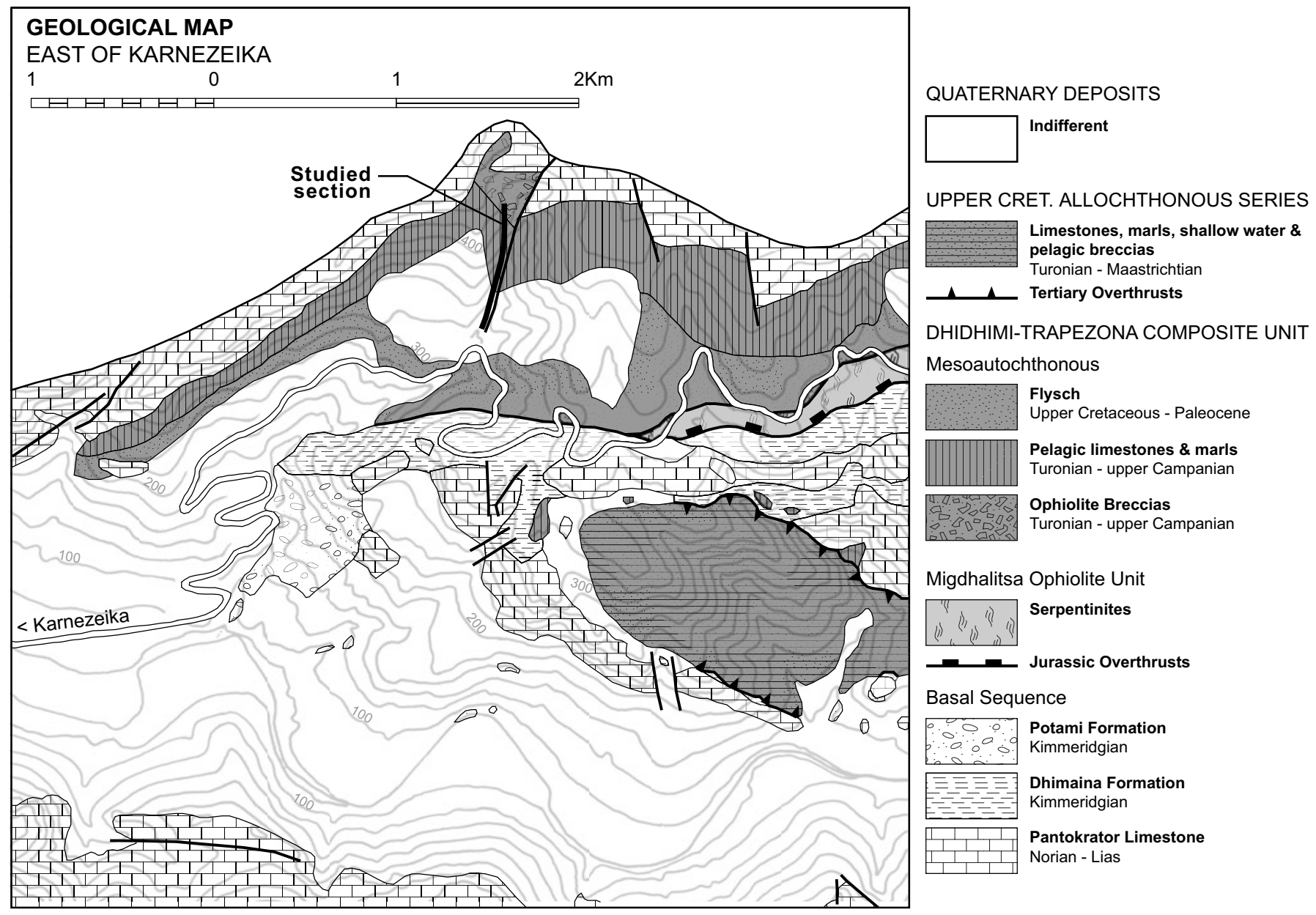

Fig. 2. Geological Map of the area east of Karnezeika (modified after Vernez 1990)

scribed above. It is a $16 \mathrm{~m}$ thick alternation of marls and red radiolarian cherts with detrital levels evolving progressively into a pelagic limestone rich in Globotruncanidae. Up section follow $10 \mathrm{~m}$ of disorganised boulder breccias with a limestone interbed, which are in turn unconformably covered by quaternary breccias.

\section{Radiolarian biochronology}

Today no standard radiolarian biochronology is available for the Late Cretaceous. However, several local and regional radiolarian zonations have been proposed in the past (see citations above).

In comparing the published range charts it becomes evident that the chronostratigraphic range of any given taxon proposed by different authors shows major discrepancies for the studied assemblage (Figs. 4a-b-c-d) from one pubication to the other. This may be due to uncertainties in the chronostratigraphic calibration of radiolarian occurrences. However, most radiolarian zonations of the Late Cretaceous are rather well calibrated by means of plaktonic foraminifera and nannofossils. We rather believe that the differences result from locally inclompete ranges of radiolarian taxa, either due to plaeobiogeographic or paleoecologic exclusions, or due to preservational (diagenetic) biases. The only way to use these radiolarian zonations is to maximise the range of each taxon by stacking the "spartial" ranges expressed in each publication. In principle, the best way of doing this is to create Unitary Associations (Guex 1977-1991: Baumgartner et al. 1980; Guex \& Davaud 1982, 1984; Baumgartner 1984 and Savary \& Guex 1991) in using the occurrence data of well-defined taxa only, detached from the chronostratigraphic calibrations (Baumgartner et al. 1995). In such a way, we can construct a range chart for the Late Cretaceous that reflects maximal ranges of each taxon with respect to the maximal ranges of all other taxa. This work is in progress, but not completed (Jackett et al. 2002 and Diserens et al. 2003). For this paper the comparison of ranges is based on the chronostratigraphic range of each taxon expessed by each author. We have simply stacked these chronostratigraphic ranges to obtain a minimum and a maximum age for the existence of each taxon. We are aware of the possible errors that may arise 


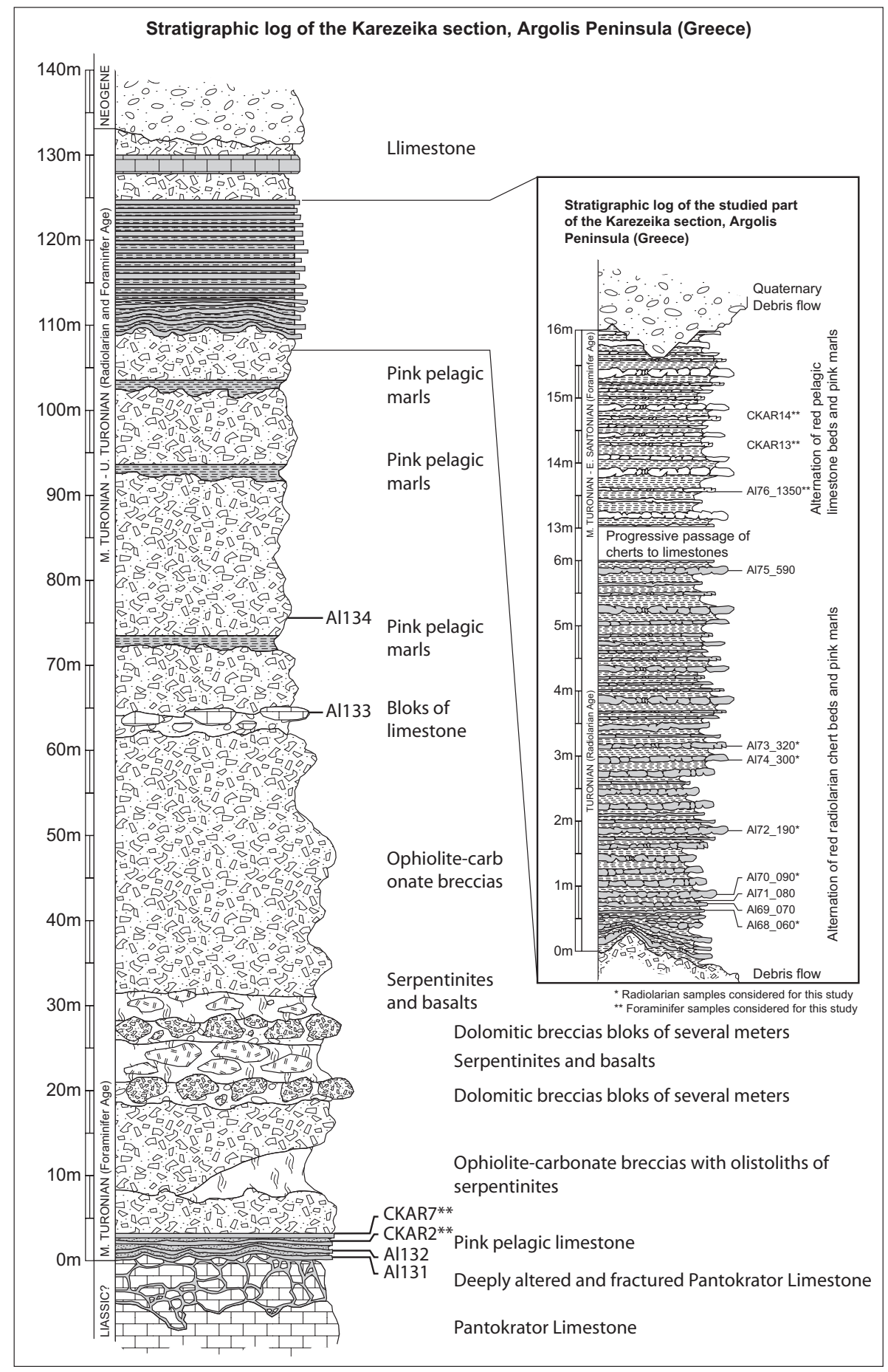

Fig. 3. Stratigraphic log of the Karnezeika section. The inset at right shows the pelagic interval studied for radiolarians and planktonic foraminfera with the samples. using this procedure. We are, however, in good company, since this procedure was practiced in a series of papers on Mesozoic Radiolaria (e.g. De Wever et al. 1986).

Despite the major discrepancies between the ranges of each author, a Turonian age can be stated using the procedure described above. This age is mainly based on the presence of Patellula ecliptica, Patellula heroica, Praeconocaryomma cali- forniaensis and Afens liriodes (have not been cited from earlier than early Turonian), found together with Pseudoaulophacus putahensis (has not been cited from later than late Turonian). Moreover, Dictyomitra urakawensis, Stichomitra communis and Pseudodictyomitra pseudomacrocephala have not been cited from later than Coniacian, Crucella messinae have not been cited from later than early Santonian (Fig. 5). 


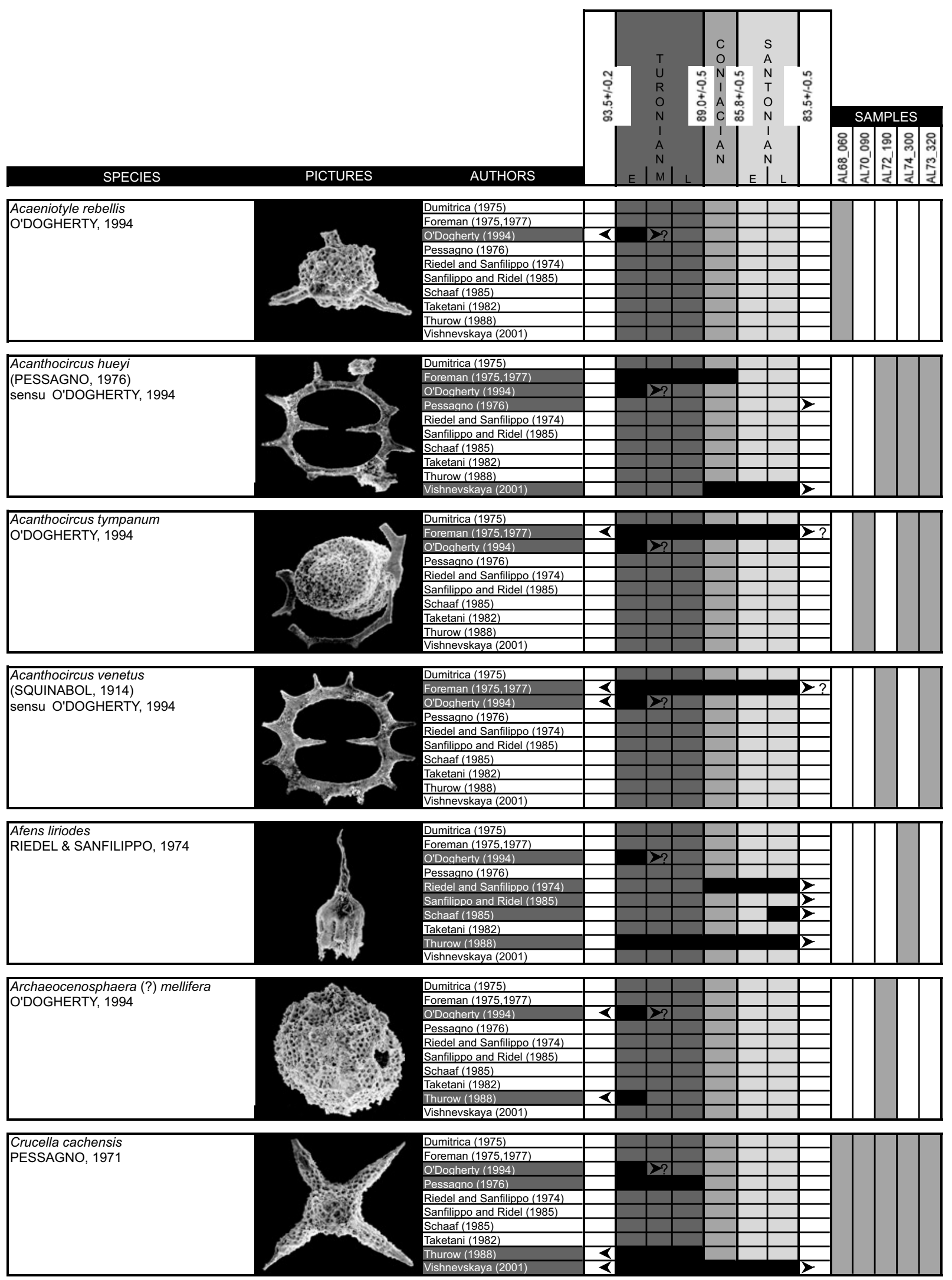

Fig. 4a. Selected Turonian-Santonian radiolarian ranges according to 10 authors as cited in the figures. Note the major discrepencies between authors. 


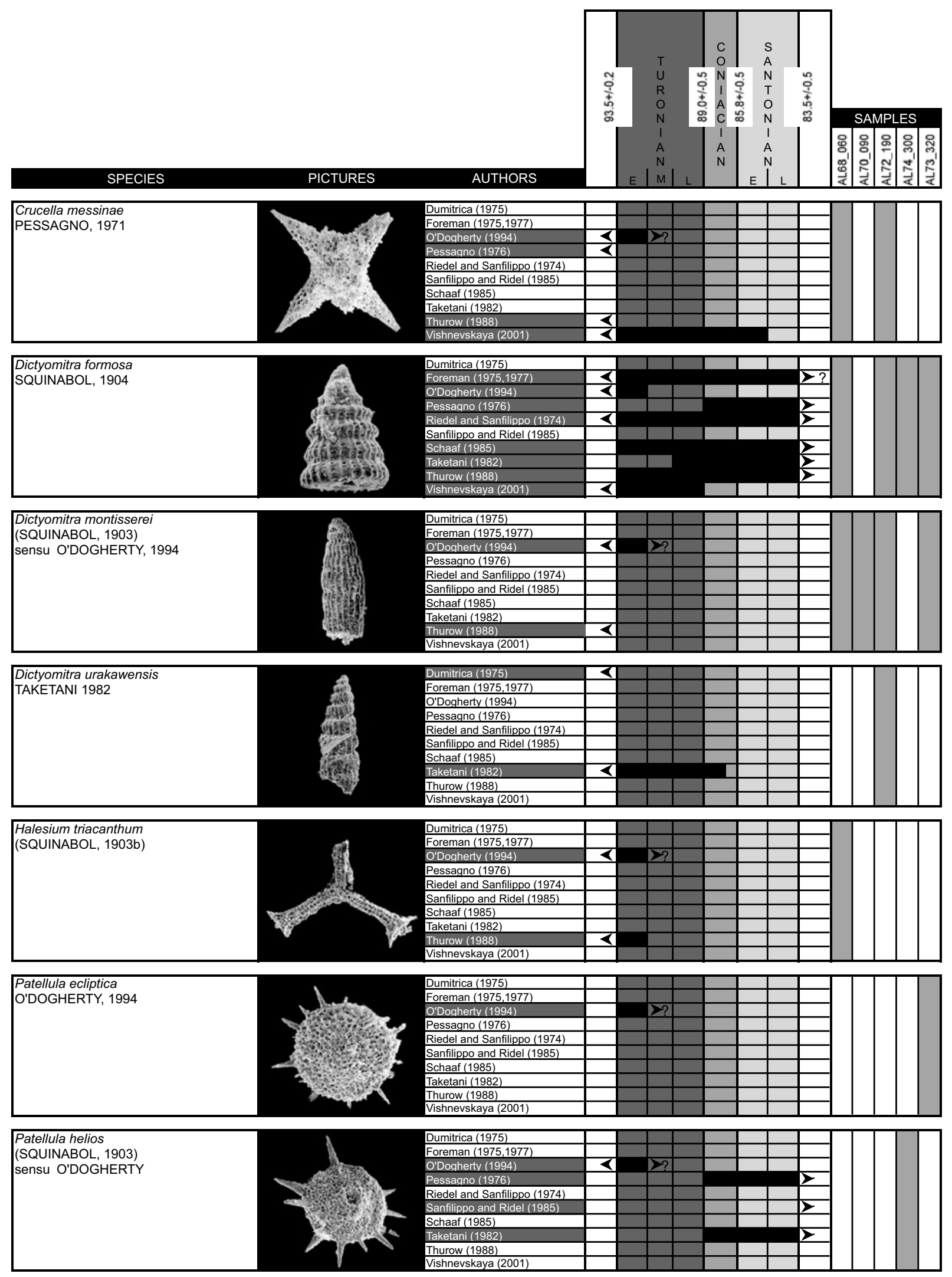

Fig. 4b. Selected Turonian-Santonian radiolarian ranges according to 10 authors as cited in the figures. Note the major discrepencies between authors. 


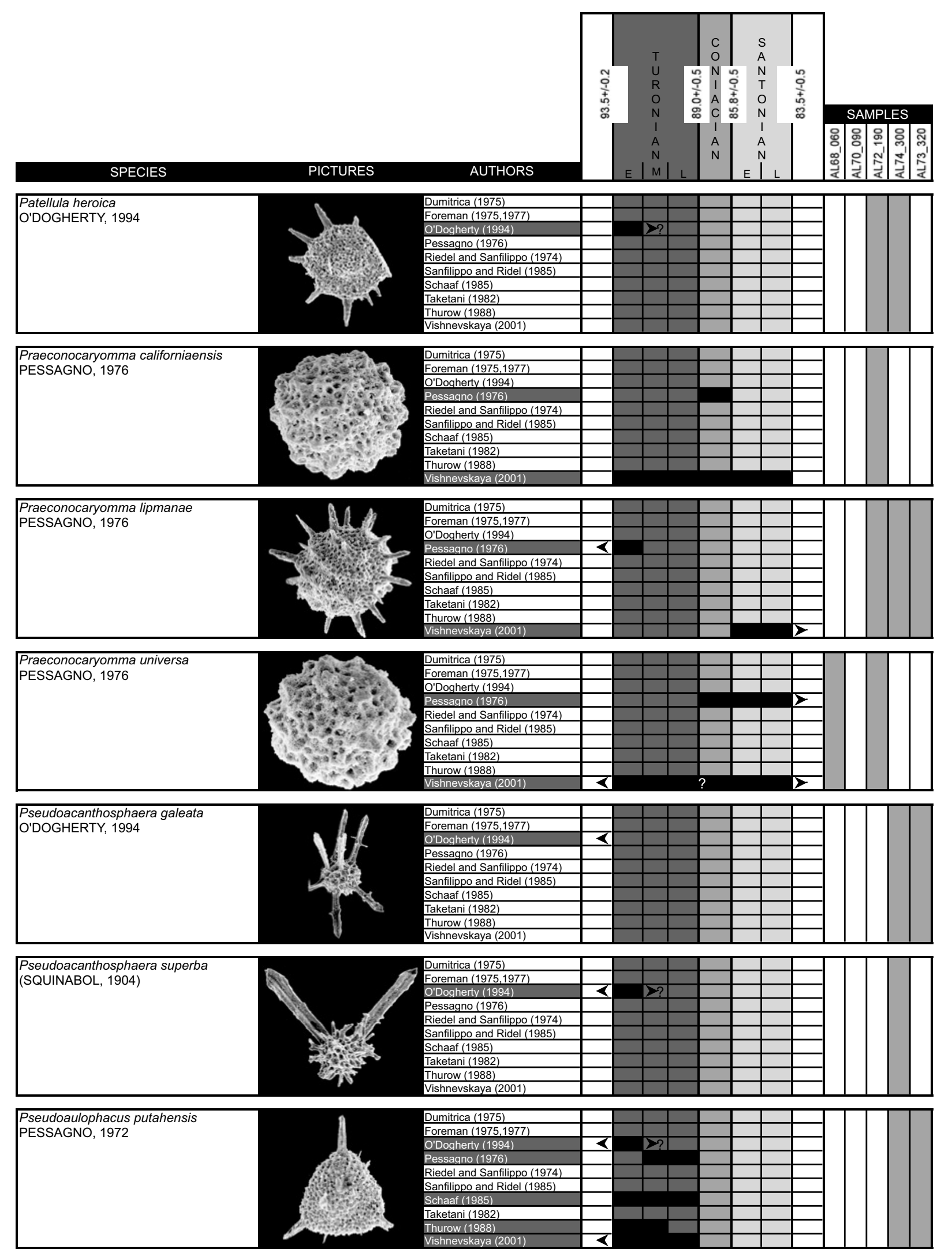

Fig. 4c. Selected Turonian-Santonian radiolarian ranges according to 10 authors as cited in the figures. Note the major discrepencies between authors. 
Pseudoaulophacus sculptus

(SQUINABOL, 1904)

sensu O'DOGHERTY, 1994

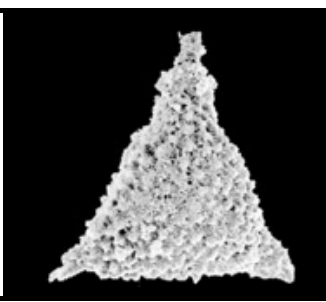

Dumitrica (1975)

Foreman $(1975,1977)$

O'Dogherty (1994)

Pessagno (1976)

Riedel and Sanfilippo (1974)

Sanfilippo and Ridel (1985)

Schaaf (1985)

Taketani (1982)

Thurow (1988)

Vishnevskaya (2001)
Pseudodictyomitra

pseudomacrocephala

(SQUINABOL, 1903)

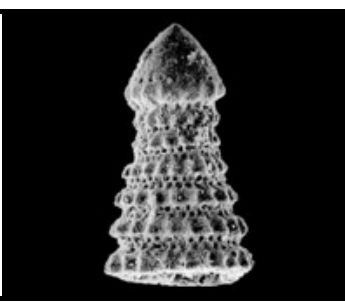

Dumitrica (1975)

Foreman $(1975,1977)$

O'Dogherty (1994)

Pessagno (1976)

Riedel and Sanfilippo (1974

Sanfilippo and Ridel (1985)

Schaaf (1985)

Taketani (1982)

Thurow (1988)

Vishnevskaya (2001)
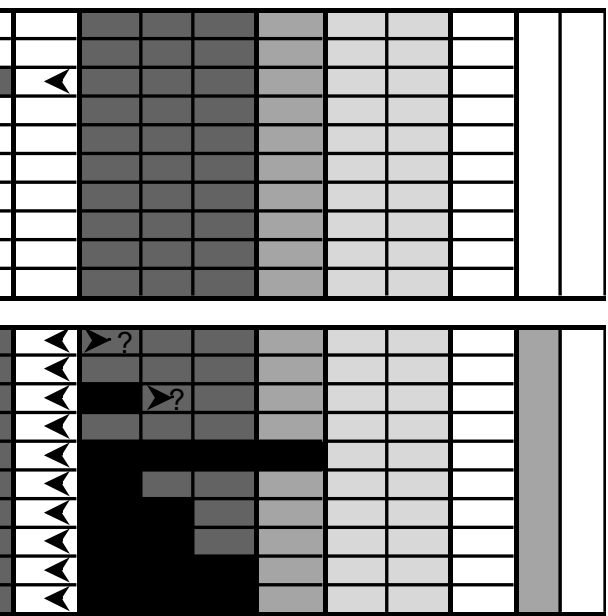

Stichomitra communis

SQUINABOL, 1903

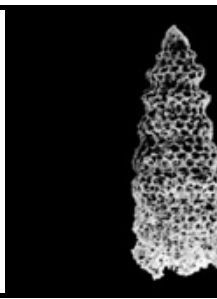

\begin{tabular}{l|l}
\hline Dumitrica (1975) & \} $\\
{\hline \text { Foreman (1975,1977) }} &{ } \\
{\hline \text { O'Dogherty (1994) }} &{ } \\
{\hline \text { Pessagno (1976) }} &{ } \\
{\hline \text { Riedel and Sanfilippo (1974) }} &{ } \\
{\hline \text { Sanfilippo and Ridel (1985) }} &{ } \\
{\hline \text { Schaaf (1985) }} &{ } \\
{\hline \text { Taketani (1982) }} &{ } \\
{\hline \text { Thurow (1988) }} &{ } \\
{\hline \text { Vishnevskaya (2001) }} &{<} \\
{\hline}$
\end{tabular}

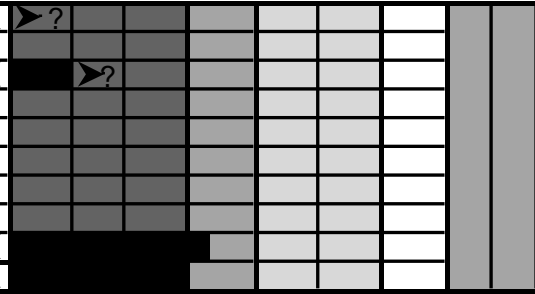

Stichomitra stocki

(CAMPBELL \& CLARK, 1944)

sensu O'DOGHERTY, 1994
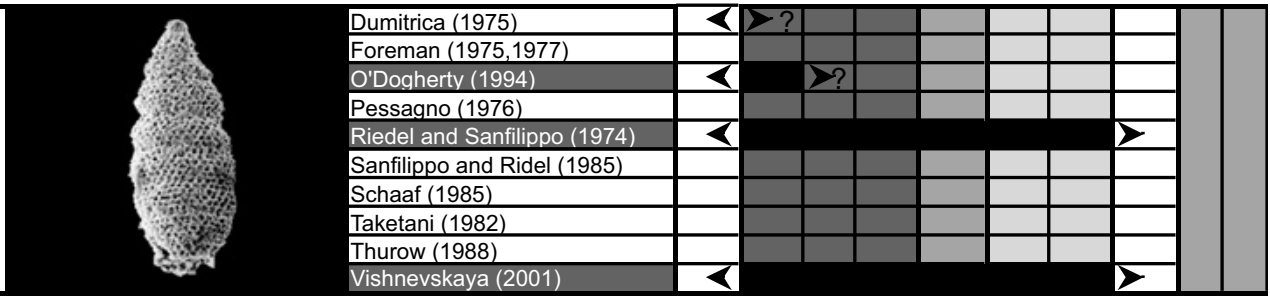

Tetracanthellipsis euganeus

SQUINABOL, 1903
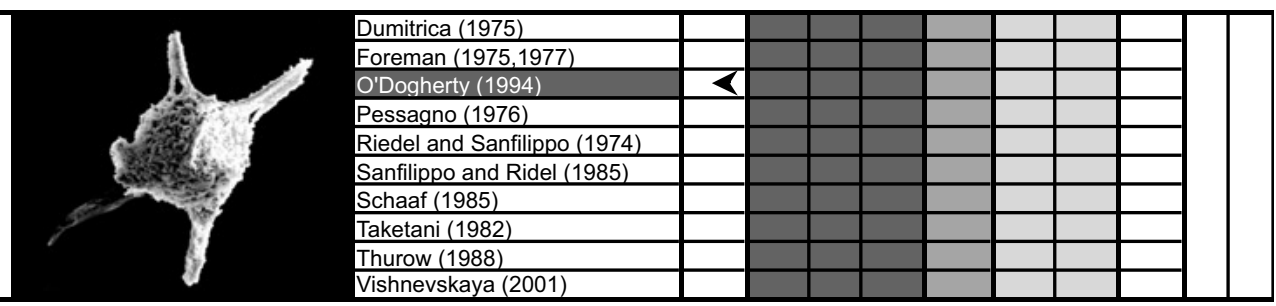

\section{Triactoma cellulosa}

FOREMAN, 1973
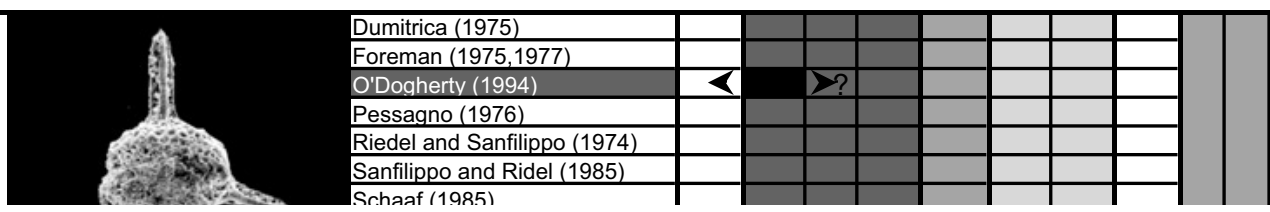

Fig. 4d. Selected Turonian-Santonian radiolarian ranges according to 10 authors as cited in the figures. Note the major discrepencies between authors. 


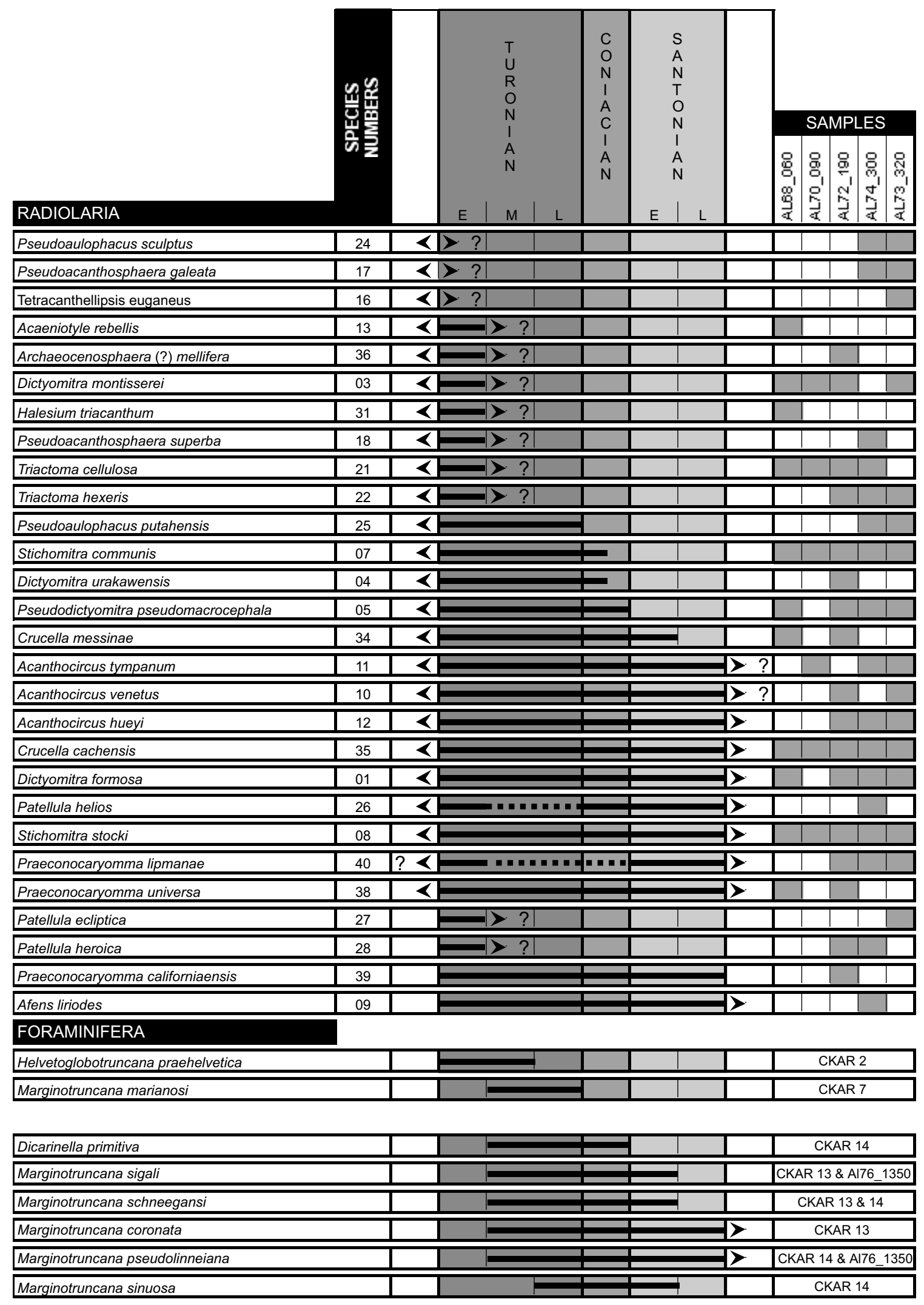

Fig. 5. Summary of Turonian to Santonian radiolarian and foraminfer ages obtained for the studied samples. The radiolarian ranges represent maximum ranges according to the 10 authors cited in figure 4. 


\section{Systematic Paleontology of Radiolaria}

The suprageneric systematics presented here follow De Wever et al. (2001). The synonymies given include the original description of each taxon and additional synonymies from the following publications: Dumitrica (1975), Foreman (1975, 1977), O'Dogherty (1994), Pessagno (1976), Riedel \& Sanfilippo (1974), Sanfilippo \& Riedel (1985), Schaaf (1985), Taketani (1982), Thurow (1988), Vishnevskaya (2001). For further synonymies the reader is refered to these publications.

\section{Class ACTINOPODA}

Subclass RADIOLARIA MÜlLER 1858

Superorder POLYCYSTINA EHRENBERG 1838

Order SPUMELLARIA EHRENBERG 1875

Superfamily ACTINOMMACEA (A) HAECKEL 1862, emend.

DE WeVER et al. 2001

Family PAR VIVACCIDAE Pessagno \& Yang in Pessagno et al. 1989

Subfamily ACAENIOTYLINAE YANG 1993

\section{Genus ACAENIOTYLE Foreman 1973}

\section{Acaeniotyle rebellis O'DOGHERTY 1994}

(Plate 1, Figs. 21-22)

1994 Acaeniotyle rebellis n. sp. O'DOGHERTY, p. 287-288, pl. 51, figs. 5-10.

\section{Acaeniotyle sp. A}

(Plate 1, Fig. 23)

Remarks. - Test with two primary spines well preserved and at the bottom right part of the shell a third spine. The angle between the three primary spines is 90 degrees (perhaps it had originally four primary spines?).

\section{Acaeniotyle sp. $B$}

(Plate 1, Fig. 24)

Remarks. - Specimen not very well preserved with only one spine. Test with tubercles.

\section{Family XIPHOSTYLIDAE HAECKEL 1881}

Genus ARCHAEOCENOSPHAERA Pessagno \& YANg in PESSAGNO et al. 1989

\section{Archaeocenosphaera (?) mellifera O'DOGHERTY 1994}

(Plate 2, Fig. 18-19)

1988 Hemicryptocapsa polyhedra DumitriCA. - ThuRow,, p. 401, pl. 1, fig. 1.

1988 Hemicryptocapsa sp. cf. H. polyhedra Dumitrica. - ThUrow, p. 401, pl. 5 , fig. 2.

1994 Archaeocenosphaera? mellifera n. sp. O’DOGHERTY, p. 375-376, pl. 74, figs. $1-5$.
Archaeocenosphaera (?) sp.

(Plate 2, Figs. 20-21)

Remarks. - Cortical shell very large, spherical with symmetrical meshwork. Cortical shell with very large polygonal pores. A medullary spongy cortical shell can be seen through the large pores of the first one. Very long spines occur at pore junctions. Spines with circular cross-section.

\section{Genus TRIACTOMA RÜsT 1885}

Triactoma cellulosa FOREMAN 1973

(Plate 1, Figs. 30-31)

1973 Triactoma cellulosa new species Foreman, p. 259, pl. 2, figs 9-10; pl. 16, fig. 9.

1994 Triactoma cellulosa ForemAn. - O'DOGHERTY, p. 300-301, pl. 54, figs $19-23$.

Triactoma hexeris O'DOGHERTY 1994

(Plate 1, Figs. 32-33)

1994 Triactoma hexeris n. sp. O’DogherTy, p. 303, pl. 55, figs. 14-21.

Triactoma sp. aff. T. hexeris O'DOGHERTY 1994

(Plate 1, Fig. 34))

Remarks. - Test with cortical shell less rouded then previous species, but more hexagonal thiner. Pores are not clearly hexagonal.

Superfamily ACTINOMMACEA (B) HAECKel 1862, emend. DE WEVER et al. 2001

Family ACTINOMMIDAE HAECKEL 1862

Genus PSEUDOACANTHOSPHAERA O'DOGHERTY 1994

Pseudoacanthosphaera galeata O'DOGHERTY 1994

(Plate 1, Fig. 26)

1994 Pseudoacanthosphaera galeata n. sp. O'DogherTy, p. 297, pl. 53, figs. 16-19.

Pseudoacanthosphaera superba (SQUINABOL 1904) (Plate 1, Fig. 27)

1904 Trisphaera superba n. sp. SQUinABOL, p. 190, pl. 2, fig. 13.

1994 Pseudoacanthosphaera superba (SQUINABOL). - O'Dogherty, p. 298-299, pl. 54, figs. 5-10.

Pseudoacanthosphaera sp. aff. P. spinosissima (SQUINABOL 1904)

(Plate 1, Fig. 28)

Remarks. - Test with a small spinose cortical shell and two long three-bladed primary spines. 
Pseudoacanthosphaera (?) sp.

(Plate 1, Fig. 29)

Remarks. - Test with ellipsoidal cortical shell and three, maybe four primary spines (the fourth one is not visible). Primary spines three-bladed. Meshwork developing small secondary spines at pore vertices.

\section{Genus TETRACANTHELLIPSIS SquINABOL 1903}

Tetracanthellipsis euganeus SQUINABOL 1903

(Plate 1, Fig. 25)

1903 Tetracanthellipsis euganeus n. sp. SouInABOL, p. 117, pl. 8, fig. 9.

1994 Tetracanthellipsis euganeus Souinabol. - O'DogherTy, p. 295-296, pl. 53, figs. $8-10$.

Superfamily ACTINOMMACEA (C) HAECKEL 1862, emend. DE WEVER et al. 2001

Family CONOCARYOMMIDAE LIPMAN 1969

\section{Genus PRAECONOCARYOMMA Pessagno 1976}

\section{Praeconocaryomma universa PESSAGNO 1976}

(Plate 2, Figs. 22-23)

1976 Praeconocaryomma universa n. sp. Pessagno, p. 42, pl. 6, fig. 14-16.

1982 Praeconocaryomma universa Pessagno. - TAKETANi, p. 47, pl. 1, figs. 3a-b, 4; pl. 9, fig. 4.

2001 Praeconocaryomma universa PESSAGnO. - VishneVSKAYA, p. 179, pl. 21, fig. 3; pl. 24, fig. 1; pl. 97, fig. 1; pl. 113, fig. 5; pl. 125, fig. 1-2; pl. 126, fig. 1.

2001 Praeconocaryomma? universa Pessagno. - Vishnevskaya, p. 179, pl. 21, figs. 11.

2001 Praeconocaryomma ex gr. universa Pessagno. - VishnevsKaya, p. 179, pl. 80 , figs. 4 ; pl. 81 , fig. 1 .

\section{Praeconocaryomma californiaensis PESSAGNO 1976}

(Plate 2, Figs. 24-25)

1976 Praeconocaryomma californiaensis n. sp. Pessagno, p. 41, pl. 7, fig. 1-8. 1982 Praeconocaryomma californiaensis PESSAGNO - TAKETANI, p. 47, pl. 1, figs. 2a-c; pl. 9, figs. 1-2.

\section{Praeconocaryomma lipmanae PESSAGNO 1976}

(Plate 2, Figs. 26-27)

1976 Praeconocaryomma lipmanae n. sp. PessAgno, p. 41-42, pl. 4, fig. 12-13.

1982 Praeconocaryomma lipmanae PeSSAgno. - TAKETAni, p. 47, pl. 9, fig. 3.

\section{Praeconocaryomma sp.}

(Plate 2, Figs. 28-29)

Remarks. - Elliptical cortical shell in outline with numerous equally spaced, cone-like mammae. Cortical shell with or without radial spines circular in cross-section projecting from center of each mamma. Cortical shell with pore frames of uniform size. Cortical shell with about ten elliptical pore frames on each mamma.

\section{Superfamily PYLONIACEA HAECKEL 1881 \\ Subsuperfamily DACTYLIOSPHAERILAE SQUINABOL 1904 \\ Family HAGIASTRIDAE RIEDEL 1971}

\section{Genus CRUCELLA Pessagno 1971}

Crucella messinae PESSAGNO 1971

(Plate 2, Figs. 14-15)

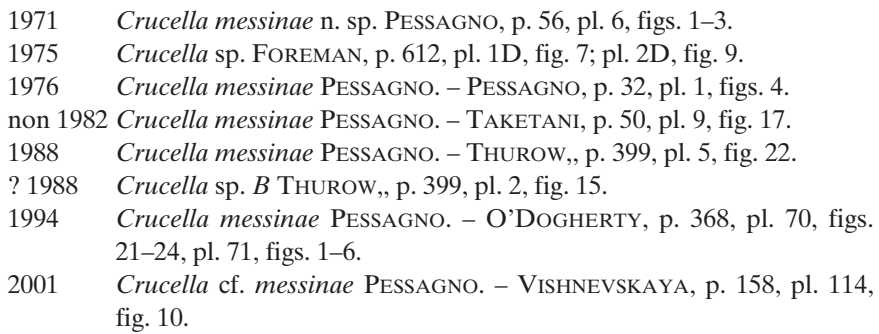

\section{Crucella cachensis PESSAGNO 1971}

(Plate 2, Figs. 16-17)

$$
\begin{aligned}
& 1971 \text { Crucella cachensis n. sp. Pessagno, p. 53, pl. 9, figs. 1-3. } \\
& 1976 \text { Crucella cachensis Pessagno. - Pessagno, p. 31, pl. 3, figs. 14-15. } \\
& 1982 \text { Crucella cachensis Pessagno. - TAKetani, p. 50, pl. 9, fig. } 16 . \\
& 1988 \text { Crucella cachensis Pessagno. - Thurow, p. 399, pl. 2, fig. } 13 . \\
& 1994 \text { Crucella cachensis Pessagno. - O’Dogherty, p. 370, pl. 71, figs. } \\
& \text { 15-22. } \\
& 2001 \text { Crucella cachensis Pessagno. - Vishnevskaya, p. 158, pl. 95, fig. 5; } \\
& \text { pl. } 125 \text {, fig. } 12 \text {; pl. 129, fig. } 3 .
\end{aligned}
$$

\section{Subsuperfamily PATULIBRACCHIILAE PEssagno 1971 Family PATULIBRACCHIIDAE Pessagno 1971}

\section{Genus HALESIUM Pessagno 1971}

Halesium triacanthum (SQUINABOL 1903) sensu O'DOGHERTY, 1994

(Plate 2, Fig. 10)

1903 Dictyastrum triacanthos n. sp. SouINABOL, p. 121, pl. 9, fig. 28

? 1988 Halesium quadratum Pessagno. - Thurow,, p. 401, pl. 2, fig. 10.

1994 Halesium triacanthum (Souinabol). - O'DOGHERTY, p. 350-351, pl. 65, figs. 9-14.

\section{Halesium sp.}

(Plate 2, Figs. 11-12)

Remarks. - Test with 3 relatively thick and small rays.

\section{Genus PESSA GNOBRACCHIA Kozur \& Mostler 1978}

\section{Pessagnobracchia sp.}

(Plate 2, Fig. 13) 
Remarks. - Spongy three-rayed test with irregular arrangement of pores on rays.

Family PSEUDOAULOPHACIDAE RIEDEL 1967

Subfamily PSEUDOAULOPHACINAE RIEDEL 1967

Genus DACTILYODISCUS SQUINABOL 1903

\section{Dactyliodiscus sp.}

(Plate 2, Figs. 8-9)

Remarks. - Test is disc-shaped and circular in outline with a variable number of equatorial spines. Meshwork spongy with irregularly pore frames. Poorly defined central raised area. Upper and lower surfaces of the test with small tubercles.

\section{Genus PSEUdOAULOPHACUS PESSAgNo 1963}

Pseudoaulophacus sculptus (SQUINABOL 1904) sensu O'DOGHERTY 1994

(Plate 1, Figs. 35-36)

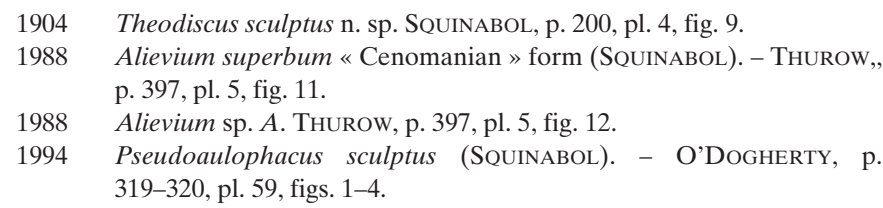

Pseudoaulophacus putahensis PESSAGNO 1972

(Plate 1, Figs. 37-38)

1972 Pseudoaulophacus putahensis n. sp. PessAgno, p. 301, pl. 27, fig. 1.

1976 Pseudoaulophacus putahensis Pessagno. - Pessagno, p. 28, pl. 3, fig. 13.

1988 Pseudoaulophacus putahensis Pessagno. - Thurow, p. 404, pl. 2, fig. 4.

1994 Pseudoaulophacus putahensis Pessagno. - O’Dogherty, p. 320-321, pl. 59, figs. 5-13.

2001 Pseudoaulophacus putahensis PESSAGNO. - VishneVSKAYA, p. 181, pl. 130, fig. 7.

Superfamily SPONGURACEA HAECKEL 1862

Family SPONGURIDAE HAECKEL 1862

\section{Genus PATELLULA Kozlova 1972}

Patellula helios (SQUINABOL 1903) sensu O'DOGHERTY 1994 (Plate 2, Figs. 1-2)

1903 Stylotrochus helios n. sp. Souinabol, p. 124, pl. 10, figs. 23-23a.

1976 Pseudoaulophacus lenticulatus (WHITE). - PessAGno, p. 28, pl. 9, figs. 11-12.

1982 Pseudoaulophacus lenticulatus (WHITE). - TAKETANI, p. 51, pl. 10, fig. 11.

1985 Pseudoaulophacus lenticulatus (WHITE). - SANFILIPPO \& RIEDEL, p. 596, text-figs. $6.4 \mathrm{a}-\mathrm{b}$.

1994 Patellula helios (Souinabol). - O’Dogherty, p. 327-328, pl. 60, figs. 19-24.
Patellula ecliptica O'DOGHERTY 1994

(Plate 2, Figs. 3-4)

1994 Patellula ecliptica n. sp. O'DogherTy, p. 329, pl. 61, figs. 1-5.

Patellula heroica O'DOGHERTY 1994

(Plate 2, Figs. 5-6)

1994 Patellula heroica n. sp. O’DogherTy, p. 330, pl. 61, figs. 6-11.

Patellula sp.

(Plate 2, Fig. 7)

Remarks. - Test flattened ellipsoidal with fifteen spines radiating in the same equatorial plane.

Order ENTACTINARIA KOZUR \& MOSTLER 1982

Family SATURNALIDAE DEFLANDRE 1953

Subfamily SATURNALINAE DEFLANDRE 1953

Genus ACANTHOCIRCUS SQUINABOL 1903

Acanthocircus venetus (SQUINABOL 1914) sensu O'DOGHERTY 1994

(Plate 1, Figs. 15-16)

1914 Saturnalis venetus n. f. SQuinabol, p. 269, 299, pl. 20[1], fig. 2; pl. 24[5], fig. 1.

?1975 Spongosaturnalis horridus (SQUINABOL). - FOREMAN, p. 610, pl. 4, fig. 3 .

1975 Spongosaturnalis hueyi group (PeSSAGNO). - ForemAn, p. 611, pl. 1B, figs. 1-3; non pl. 1A, figs. 7-8 (=A. tympanum?).

1975 Spongosaturnalis (?) preclarus new species ForemAn, p. 611, pl. 1A, figs. 4-5; pl. 4, fig. 8 .

1994 Acanthocircus venetus (SQUINABOL). - O'DOGHERTY, p. 256, pl. 45 , figs. $1-8$.

\section{Acanthocircus tympanum O'DOGHERTY 1994}

(Plate 1, Figs. 17-18)

pars 1975 Spongosaturnalis hueyi group (PESSAGNo). - ForemAn, p. 611, pl. 1A, figs. 7-8; non pl. 1B, figs. 1-3 (=A. venetus?)

1994 Acanthocircus tympanum n. sp. O'Dogherty, p. 259-260, pl. 45, figs. 17-24.

Acanthocircus hueyi (PESSAGNO 1976) sensu O’DOGHERTY 1994 (Plate 1, Figs. 19-20)

1975 Spongosaturnalis hueyi (PESSAGNo). - Foreman, p. 611, pl. 1A, fig. 6; pl. 4, fig. 10 .

1976 Spongosaturnalis hueyi n. sp. Pessagno, p. 39, pl. 12, fig. 1.

1994 Acanthocircus hueyi (Pessagno). - O’Dogherty, p. 260-261, pl. 46, figs. 1-5.

2001 Spongosaturnalis hueyi (PESSAgno). - VishneVsKayA, p. 186, pl. 122 , fig. 2.

2001 Spongosaturnalis ex. gr. hueyi (PESSAGNo). - VishnEVSKAYA, p. 186, pl. 92, figs. 7-8, pl. 95, figs. 1-3.

Order NASSELARIA EHRENBERG 1875

Superfamily ARCHAEDICTYOMITRACEA PESSAGNO 1976 
Family ARCHAEDICTYOMITRIDAE PESSAGNO 1976

\section{Genus DICTYOMITRA ZITTEL 1876}

Dictyomitra formosa SQUINABOL 1904

(Plate 1, Figs. 1-2)

1904 Dictyomitra formosa n. sp. Souinabol, p. 232, pl. 10, fig. 4.

1974 Dictyomitra torquata Foreman. - Riedel \& SANFILIPPO, p. 778, pl. 5, figs. 1,2 and 4 .

1975 Dictyomitra duodecimcostata (SQuinabol). - Foreman, p. 614, pl. 7, fig. 8; pl. 1G, fig. 5 .

1976 Dictyomitra formosa Souinabol. - Pessagno, p. 51, pl. 8, figs. 10-12.

1982 Dictyomitra formosa Souinabol. - TAKETANI, p. 58, pl. 4, figs. 6a-b; pl. 11, fig. 13.

1985 Dictyomitra formosa SouinaBol. - SCHAAF,, text-fig.11, p. 250.

1988 Dictyomitra formosa Souinabol. - Thurow,, p. 400, pl. 1, figs. 23 and 25.

1994 Dictyomitra formosa Squinabol. - O’Dogherty, p. 80, pl. 4, figs. 8-12.

2001 Dictyomitra formosa Souinabol. - Vishnevskaya, p. 160, pl. 25, fig. 10.

Dictyomitra sp. cf. D. formosa SQUINABOL 1904

(Plate 1, Fig. 3)

(species number 2)

Dictyomitra montisserei (SQUINABOL 1903) sensu O'DOGHERTY 1994

(Plate 1, Figs. 4-5)

1903 Stichophormis Montis Serei n. sp. SQuinabol, p. 137, pl. 8, fig. 38.

1975 Dictyomitra sp. A. Foreman, p. 615, pl. 1G, fig. 7; pl. 2G, figs. 18 and 20.

1982 Archaedictyomitra sp. A. TAKETANi, p. 58, pl. 4, figs. 5a-b.

? 1988 Archaedictyomitra lacrimula (ForEmAN). - THUROw,, p. 397, pl. 3, fig. 8.

1988 Archaedictyomitra simplex Pessagno. - Thurow, p. 398, pl. 3, fig. 9.

1994 Dictyomitra montisserei (SQUINABOL 1903b). - O'DogherTy, p. 77, pl. 3, figs. 1-29.

\section{Dictyomitra urakawensis (TAKETANI 1982)}

(Plate 1, Fig. 6)

? 1975 Dictyomitra sp. - Dumitrica,, text-fig.2; 8 .

1982 Dictyomitra urakawensis n. sp. TAKETANI, p. 59, pl. 4, figs. 8a-b; pl. 11, fig. 16.

Superfamily AMPHIPYNDACEA RIEDEL 1967

Family SPONGOCAPSULIDAE PESSAGNO1977

\section{Genus TORCULUM O’DOGHERTy 1995}

Torculum coronatum (SQUINABOL1904)

(Plate 1, Fig. 9)

1904 Theoconus coronatus n. sp. Souinabol, p. 220, pl. 8, fig. 3.

1976 Stichomitra (?) zamoraensis n. sp. Pessagno, p. 54, pl. 3, figs. 7-9.

1982 Spongocapsula (?) zamoraensis (PESSAGNO). - TAKETANI, p. 62, pl. 5, figs. $6 \mathrm{a}-\mathrm{b}$; pl. 12, figs. 12-13.

1988 Theoconus coronatus Group SQuinaboL. - THurow, p. 407, pl. 4, figs. 2.
1988 Theoconus sp. A cf. T. coronatus Squinabol. - Thurow,, p. 407, pl. 4, figs. 3-4.

2001 Spongocapsula aff. zamoraensis (Pessagno). - VishnevsKaya, p. 186, pl. 77, fig. 5; pl. 90, figs. 5-6.

Superfamily EUCYRTIDIACEA EHRENBERG 1847

Family PSEUDODICTYOMITRIDAE PESSAGNO 1977

Genus PSEUdODICTYOMITRA Pessagno 1977

Pseudodictyomitra pseudomacrocephala (SQUINABOL 1903)

(Plate 1, Figs. 7-8)

1903 Dictyomitra pseudomacrocephala n. sp. SouInABOL, p. 139, pl. 10, fig. 2.

1974 Dictyomitra macrocephala Souinabol. - RiEdel \& SANFILIPPO, p. 778 , pl. 4 , figs. $10-11$; pl. 14 , fig. 11.

1975 Dictyomitra pseudomacrocephala SQuinabol. - DumitricA, textfig. $2,19$.

1975 Dictyomitra pseudomacrocephala SQuinabol. - Foreman, p. 614, pl. 7, fig. 10.

1976 Dictyomitra (?) pseudomacrocephala SQUinABOL. - PESSAGNo, p. 53, pl. 3, figs. 2-3.

1982 Pseudodictyomitra pseudomacrocephala (SQUINABOL). - TAKETANI, p. 61 , pl. 5, figs. 4a-b; pl. 12, figs. 7-8.

1985 Pseudodictyomitra pseudomacrocephala (SoUINABOL). - SANFILIPPO \& RIEDEL, p. 608, text-figs. 10. 1a-b.

1985 Pseudodictyomitra pseudomacrocephala (SQUINABOL). - SCHAAF, text-fig.11, p. 250

1988 Pseudodictyomitra pseudomacrocephala (SQUINABOL). - THUROW,, p. 405 , pl. 1, fig. 13 ; pl. 3, figs. 11-16.

1994 Pseudodictyomitra pseudomacrocephala (SQUINABOL). - O'DOGHERTY, p. 108-109, pl. 8, figs. 5-8.

2001 Pseudodictyomitra pseudomacrocephala (SQUINABOL). - VishNEVSKAYA, p. $183-184$, pl. 20, fig. 6; pl. 24, fig. 10; pl. 100, fig. 3; pl. 129, figs. 5, 9 and 10 .

\section{Family EUCYRTIDIIAE EHRENBERG 1847}

\section{Genus STICHOMITRA CAYEux 1897}

Stichomitra communis SQUINABOL 1903

(Plate 1, Figs. 10-11)

1903 Stichomitra communis n. sp. Souinabol, p. 141, pl. 8, fig. 40.

1975 Stichomitra sp. DuMITRICA,, text-fig.2, 21.

1975 Stichomitra spp. cf. D. tekschaensis AliEv. - Foreman, p. 615, pl. 2H, fig1.

1982 Stichomitra communis Souinabol. - TAKETANI, p. 54, pl. 3, fig. 9; pl. 11 , fig. 5 .

1988 Stichomitra communis SQUINABOL. - THurow,, p. 406, pl. 4, fig. 10.

1988 Stichomitra sp. cf. S. communis Souinabol. - Thurow,, p. 406, pl. 4, fig. 9.

1994 Stichomitra communis SouinABol. - O’Dogherty, p. 144-145, pl. 17, figs. 6-16.

2001 Stichomitra communis Souinabol. - VishneVsKayA, p. 188, pl. 23, fig. 8 ; pl. 79 , fig. 3 ; pl. 129 , fig. 8 .

Stichomitra stocki (CAMPBELL \& CLARK 1944) sensu O'DOGHERTY 1994

(Plate 1, Figs. 12-13)

1944 Stichocapsa (?) stocki n. sp. CAMPBELl \& CLARK, p. 44, pl. 8, figs. 31-33. 
Amphipyndax stocki (CAMPBell \& ClARK). - Riedel \& SANFILIPPO, p. 775 , pl. 15 , fig. 11 ; pl. 11, figs. $1-3$.

1975 Amphipyndax stocki (CAmpbell \& Clark). - Dumitrica, textfig.2.23.

1982 Amphipyndax stocki (CAMPBell \& ClARK). - TAKeTANi, p. 52, pl. 2, figs. 9a-b; pl. 10, figs. 13-14.

1982 Amphipyndax sp. TAKetAni, p. 52, pl. 10, fig. 16.

1988 Stichomitra (?) sp. A. Thurow,, p. 406, pl. 1, fig. 17.

1994 Stichomitra stocki (CAMPBELl \& ClARK). O'DogherTy, p. 147-148 and 150, pl. 18, figs. 9-15.

2001 Amphipyndax stocki (CAMPBELl \& ClARK). - VishneVsKayA, p. 146, pl. 1, fig. 13; pl. 4, fig. 11-13; pl. 6, fig. 12; pl. 16, fig. 1; pl. 93, fig. 4-5; pl. 94, fig. 8 and 10; pl. 99, fig. 1-3 and 8; pl. 114, fig. 12 .

2001 Amphipyndax stocki (CAMPBELl \& ClARK) var. A VishnevsKAyA. VISHNEVSKAYA, p. 146-147, pl. 16, fig. 2-6; pl. 26, fig. 6; pl. 100, fig. 4; pl. 123, fig. 16-21 and 23.

2001 Amphipyndax stocki (CAMPBell \& Clark) var. B VishnevsKaya. VishneVSKAYA, p147, pl. 3, fig. 6; pl. 12, fig. 3 and 5; pl. 15, fig. 1-5.

2001 Amphipyndax stocki (CAMPBELl \& ClARK) var. C VishnevsKAyA. VishneVsKAYA, p. 147, pl. 3, fig. 2 and 4; pl. 14, fig. 1-3.V

\section{NASSELLARIA INCERTAE SEDIS}

\section{Genus AFENS Riedel \& SANFILIPPo 1974}

\section{Afens liriodes RIEDEL \& SANFILIPPO 1974}

(Plate 1, Fig. 14)

1974 Afens liriodes new genus and new species RiEdel \& SANFILIPPO, p. 775, pl. 11, fig. 11; pl. 13, figs. 14-16.

1985 Afens liriodes Riedel \& SANFILIPPO. - SANFILIPPo \& Riedel, p. 624, text-figs. 13.3a-c.

1985 Afens liriodes Riedel \& SANFilipPo. - SCHAAF, text-fig.11, p. 250.

1988 Afens liriodes Riedel \& SANFilipPo. - ThUROw,, pl. 2,fig.1.

1994 Afens liriodes Riedel \& SANFilipPo. - O’Dogherty, p. 246, pl. 42, figs. 23-26.

\section{Planktonic Foraminifera}

In the following, we use the ranges proposed by Caron (1985). The first pelagic facies at the top of the Pantokrator Limestone is dated as middle Turonian by the presence of Helvetoglobotruncana helvetica (Pl. 3, Fig. 1, early Turonian - middle Turonian) and Marginoglobotruncana marianosi (Pl. 3, Fig. 2, middle Turonian - late Turonian).

In the pelagic limestone rich in Globotruncanidae at the top of the radiolarian cherts, the presence of Dicarinella primitiva (Pl. 3, Fig. 9, middle Turonian - Coniacian), Marginotruncana sigali (P1. 3, Figs. 5 and 12, middle Turonian - early Santonian), M. schneegansi (P1. 3, Figs. 3 and 7, middle Turonian - early Santonian), M. coronata (Pl. 3, Fig. 4, middle Turonian - early Campanian), M. pseudolinneiana (Pl. 3, Figs. 6, 10 and 11, middle Turonian - early Campanian) and M. sinuosa (Pl. 3, Fig. 8, late Turonian - early Santonian) allow to state a late Turonian to early Santonian age without further precision.

\section{Discussion and Conclusions}

The radiolarian chronostratigraphic ranges used for this paper are based on the comparison of taxon ranges established by 10 different authors (see list in introduction). By stacking chronostratigraphic ranges, we obtain a maximum range for the existence of each taxon. Despite possible inaccuracies of calibration, the radiolarian age given by this procedure is consistent with the age based on planktonic foraminifera (late Turonian to Coniacian). In combining the radiolarian and the planktonic foraminifer ages, the samples would be restricted to the late Turonian. However, the major discrepancies of published radiolarian ranges call for an urgent, major revision of the Late Cretaceous radiolarian biochronology, a project that is underway (Jackett et al. 2002 and Diserens et al. 2003). The integration of planktonic foraminifera with radiolarians may greatly enhance biochronologic resolution in sections where both groups occur.

This is the first time that Late Cretaceous radiolarians are described from the Argolis Peninsula.

\section{Acknowledgements}

The field work in the Argolis Peninsula was partly financed by the Sociéte Académique Vaudoise and the University of Lausanne, Switzerland. The salaries are paid by the Swiss National Science Foundation (grant $n^{\circ} 2000$ 063762 and $\mathrm{n}^{\circ}$ 200021-105845). We are thankful for the loan of the Vernez' thin sections by the Musée Cantonal de Géologie, Lausanne, Switzerland. We are grateful for help in the field by France Girault and Thierry Glassey; assistance in the laboratory by Sébastien Bruchez, Marc-Olivier Diserens and Sarah-Jane Jackett; and for discussions with Paulian Dumitrica. The manuscript has benefited from careful reviews by Atsushi Matsuoka and Luis O'Dogherty.

\section{SPECIES LIST}

Acaeniotyle rebellis O'DOGHERTY 1994 (Pl. 1, Figs. 1-2)

Acaeniotyle sp. A (Pl. 1, Fig. 3)

Acaeniotyle sp. B (Pl. 1, Fig. 4)

Acanthocircus hueyi ( PESSAGNO 1976) sensu O'DOGHERTY 1994 (P1. 2, Figs.18-19)

Acanthocircus tympanum O'DOGHERTY 1994 (Pl. 2, Figs. 16-17)

Acanthocircus venetus (SQUINABOL 1914) sensu O'DOGHERTY 1994 (Pl. 2, Figs. 14-15)

Afens liriodes RiEdEL \& SANFILIPPO 1974 (Pl. 2, Fig. 33)

Archaeocenosphaera (?) mellifera O'DOGHERTY 1994 (Pl. 1, Figs. 5-6)

Archaeocenosphaera (?) sp. (Pl. 1, Figs. 7-8)

Crucella cachensis PESSAGNO 1971 (Pl. 1, Figs. 29-30)

Crucella messinae PeSSAGNO 1971 (Pl. 1, Figs. 27-28)

Dactyliodiscus sp. (Pl. 2, Figs. 1-2)

Dictyomitra formosa SouinaBOL 1904 (Pl. 2, Figs. 20-21)

Dictyomitra montisserei (SQUINABOL 1903) sensu O'DOGHERTY 1994 (Pl. 2, Figs. 23-24) 
Dictyomitra sp. cf. D. formosa SQuinabol 1904 (Pl. 2, Fig. 22)

Dictyomitra urakawensis TAKETANI 1982 (Pl. 2, Fig. 25)

Halesium sp. (Pl. 1, Figs. 32-33)

Halesium triacanthum (SQUINABOL 1903) sensu O'DOGHERTY

1994 (Pl. 1, Fig. 31)

Patellula ecliptica O'DOGHeRTY 1994 (Pl. 2, Figs. 9-10)

Patellula helios (SQUINABOL 1903) sensu O'DOGHERTY 1994

(Pl. 2, Figs. 7-8)

Patellula heroica O'DOGHERTY 1994 (Pl. 2, Figs. 11-12)

Patellula sp. (Pl. 2, Fig. 13)

Pessagnobrachia sp. (Pl. 1, Fig. 34)

Praeconocaryomma californiaensis PESSAGNO 1976 (Pl. 1,

Figs. 21-22)

Praeconocaryomma lipmanae PESSAGno 1976 (Pl. 1, Figs. 23-24)

Praeconocaryomma sp. (Pl. 1, Figs. 25-26)

Praeconocaryomma universa PESSAGNO 1976 (Pl. 1, Figs.

19-20)

Pseudoacanthosphaera (?) sp. (Pl. 1, Fig.17)

Pseudoacanthosphaera galeata O'DOGHERTY 1994 (Pl. 1,

Fig. 14)

Pseudoacanthosphaera sp. aff. $\boldsymbol{P}$. spinosissima (SOUINABOL 1904) (Pl. 1, Fig. 16)

Pseudoacanthosphaera superba (SQUINABOL 1904) (Pl. 1,

Fig. 15)

Pseudoaulophacus putahensis PESSAGNO 1972 (Pl. 2, Figs. 5-6) Pseudoaulophacus sculptus (SQUINABOL 1904) sensu O'DoGHerTy 1994 (Pl. 2, Figs. 3-4)

Pseudodictyomitra pseudomacrocephala (SQUINABOL 1903)

(Pl. 2, Figs. 27-28)

Stichomitra communis SQUINABOL 1903 (Pl. 2, Figs. 29-30)

Stichomitra stocki (CAMPBELL \& CLARK 1944) sensu O'DOGHERTY 1994 (Pl. 2, Figs. 31-32)

Tetracanthellipsis euganeus SQUINABOL 1903 (Pl. 1, Fig. 18)

Torculum coronatum (SQUINABOL 1904) (Pl. 2, Fig. 26)

Triactoma cellulosa FOREMAN 1973 (Pl. 1, Figs. 9-10)

Triactoma hexeris O'DOGHERTY 1994 (Pl. 1, Figs. 11-12)

Triactoma sp. aff. T. hexeris O’DOGHERTY 1994 (Pl. 1, Fig. 13)

\section{REFERENCES}

BANDINI, A. 2004: Sédimentologie et tectonostratigraphie du sud de la péninsule de l'Argolide, Péloponèse (Grèce) \& Upper Cretaceous radiolarians of Karnezeika, (Argolis Peninsula, Peloponesus (Greece). Unpublished diploma thesis, University of Lausanne.

BAUMGARTNER, P.O. 1984: Comparison of unitary associations and probabilistic ranking and scaling as applied to Mesozoic radiolarians. Computers and Geosciences, 10 (1), 167-183.

Baumgartner, P.O. 1985: Jurassic sedimentary evolution and nappe emplacement in the Argolis peninsula (Peloponnesus, Greece). Denkschriften der Schweizerischen Naturforschenden Gesellschaft; 99, 111p. Birkhäuser, Basel, Boston, Stuttgart.

Baumgartner, P.O., De Wever, P. \& Kocher, R. 1980: Correlation of Tethyan Late Jurassic-Early Cretaceous radiolarian events. Cahiers de Micropaléontologie, 2, 23-86.
Baumgartner, P.O., GueX, J. \& Dumitrica, P. 1995: Concepts of the systematic and biostratigraphic work. In: BAUMgARTNER, P.O et al. (Eds.): Middle Jurassic to Lower Cretaceous Radiolaria of Tethys: Occurrences, Systematics, Biochronology, 11-15. Mémoires de Géologie (Lausanne), 23. Institut de Géologie et de Paléontologie, Université de Lausanne.

CAMPBELL, A.S. \& ClARK, B.L. 1944: Radiolaria from Upper Cretaceous of Middle California. Geological Society of America Special Paper, 57, 1-61.

CARon, M. 1985: Cretaceous planktic foraminifera. In: Bolli, H.M., Sauders, J.B. \& Perch-Nielsen, K. (Eds.): Plankton stratigraphy, 17-86. Cambridge University Press, Cambridge/ New York.

De Wever, P. \& Origlia-Devos, I. 1982: Datations nouvelles par les Radiolaires de la série des Radiolarites s. l. du Pinde-Olonos, (Grèce). C. R. Acad. Sc. Paris, 294, 399-404.

De WeVer, P. \& ThiéBault, F. 1981: Les Radiolaires d'âge jurassique supérieur à crétacé supérieur dans les radiolarites du Pinde-Olonos (Presqu'île de Koroni; Péloponnèse méridional, Grèce). Géobios, 14(5), 577-609.

De Wever, P., Geyssant, J.R., Azéma, J., Devos, I., Duée, G., Manivit, H. \& VRIELYNCK, B. 1986: La coupe de Santa Anna (zone de Sciacca, Sicile): une synthèse biostratigraphique des apports des macro-, micro- et nannofosiles du Jurassique supérieur et Crétacé inférieur. Revue de Micropaléontologie, 29 (3), 141-186.

De Wever, P., Dumitrica, P., Caulet, J.P., Nigrini, C. \& Caridroit, M. 2001: Radiolarians in the sedimentary record. 533 p, Gordon \& Breach Science Publ..

Diserens, M.-O., Baumgartner, P.O. \& Dumitrica, P. 2003: Age determination of late Cretaceous radiolarites in orogenic environments: an example from accreted teeranes of southern Costa Rica. Interrad X 2003, Abstracts \& Programme, University of Lausanne, Switzerland, 49-50.

DumitricA, P. 1975: Cenomanian Radiolaria at Podul Dimbovitei, Micropaleontological guide to the Mesozoic and Tertiary of the Romanian Carpathians. In: 14th European Micropaleontological Colloquium, Romania, 87-89. Institute of Geology and Geophysics, Bucarest.

Foreman, H.P. 1973: Radiolaria from DSDP Leg 20. In: HeEzen, B.C. et al. (Eds.): Initial Reports of the Deep Sea Drilling Project, 20, 249-305. U.S. Government Printing Office, Washington, D.C.

Foreman, H.P. 1975: Radiolaria from the North Pacific, Deep Sea Drilling Project, Leg 32. In: LARSON, R.L. et al. (Eds.): Initial Reports of the Deep Sea Drilling Project, 32 (Hakodate, Japan to Honolulu, Hawaii, Aug.Oct., 1973), 579-676. U.S. Government Printing Office, Washington, D.C.

Foreman, H.P. 1977: Mesozoic Radiolaria from the Atlantic Basin and its Borderlands. In: F.M. SwAIN (Ed.): Stratigraphic Micropaleontology of Atlantic Basin and Borderlands, 305-313. Elsevier Scientific Publishing Company, Amsterdam, Netherlands.

GiRAULT, F.E. 2005: Sédimentologie et tectonostratigraphie de Deprano et sa région (Argolide - Grèce). Unpublished diploma thesis, University of Lausanne.

Glassey, T. 2005: Etude sédimentologique et tectonique d'un secteur de la région de Midhéa, en Argolide septentrionale (Grèce). Unpublished diploma thesis, University of Lausanne.

GuEX, J. 1977: Une nouvelle méthode d'analyse biochronologique. Bulletin de la Société Vaudoise des Sciences Naturelles, 73, 170-216

GuEX, J. 1979: Terminologie et méthodes de la biostratigraphie moderne: commentaires critiques et propositions. Bulletin de la Société Vaudoise des Sciences Naturelles, 74, 170-216.

Guex, J. 1980: Calcul, caractérisation et identification des associations unitaires en biochronologie. Bulletin de Société Vaudoise des Sciences Naturelles, 75, 111-126.

GuEX, J. 1981: Associations virtuelles et discontinuités dans la distribution des espèces fossiles: un exemple intéressant. Bulletin de la Société Vaudoise des Sciences Naturelles, 75, 179-197.

GuEX, J. 1984: Estimation numériques de la qualité de l'enregistrement fossile des espèces. Bulletin de la Société Vaudoise des Sciences Naturelles, 77, $79-89$.

GuEX, J. 1987: Corrélations biochronologiques et associations unitaires. 244p., Presses Polytechniques Romandes, Lausanne.

Guex, J. 1988: Utilisation des horizons maximaux résiduels en biochronologie. Bulletin de la Société Vaudoise des Sciences Naturelles, 79 (2), 135-142. 
GueX, J. 1991: Biochronological Correlations. Springer-Verlag, Berlin/Heidelberg/New York. $250 \mathrm{p}$.

GueX, J. \& Davaud, E. 1982: Recherche automatique des associations unitaires: option nouvelle et exemple d'application. Bulletin de la Société Vaudoise des Sciences Naturelles, 78 (2), 195-205.

Guex, J. \& DAvaud, E. 1984: Unitary Associations Method: use of graph theory and computer algorithm. Computers \& Geosciences, 10 (1), 69-96.

Jackett, S.-J., Diserens, M.-O \& BAumgartner, P.O. 2002: Late Cretaceous To Early Cenozoic Radiolarian Biochronology Of Low-Latitude Orogenic Regions. Problems And Solutions. Abstracts and Proceedings of the Norwegian Geological Society, 1, 63.

Neumann, P. 2003: Ablagerungsprozesse, Event- und Biostratigraphie kreidezeitlicher Tiefwassersediemente der Tethys in der Olonos-Pindos-Zone Westgriechenlands. Müncher Geowiss. Abh., 40, 1-156.

O’Dogherty, L. 1994: Biochronology and Paleontology of Mid-Cretaceous Radiolarians from Northern Apennines (Italy) and Betic Cordillera (Spain). Mémoires de Géologie (Lausanne), 21. 415p. Institut de Géologie et de Paléontologie, Université de Lausanne.

Pessagno, E.A. 1971: Jurassic and Cretaceous Hagiastridae from the BlakeBahama Basin (Site 5A, JOIDES Leg 1) and the Great Valley Sequence, California Coast Ranges. Bulletins of American Paleontology, 60(264), $5-83$.

Pessagno, E.A. 1976: Radiolarian zonation and stratigraphy of the Upper Cretaceous portion of the Great Valley Sequence, California Coast Ranges. Micropaleontology spec. Publ., 2, 1-95.

RIEDEL, W.R. \& SANFILIPPO, A. 1974: Radiolaria from the southern Indian Ocean, DSDP Leg 26. In: DAVIES, T.A., et al. (Eds.): Initial Reports of the Deep Sea Drilling Project, 26 (Durban, South Africa to Fremantle, Australia; Sept.-Oct. 1972), 771-781. U.S. Government Printing Office, Washington, D.C.

SANFILIPPO, A. \& RIEDEL, W.R. 1985: Cretaceous Radiolaria. In: Bolli, H.M., Sauders, J.B. \& Perch-Nielsen, K. (Eds.): Plankton stratigraphy, 631-712. Cambridge University Press, Cambridge/ New York.

SAVARY, J. \& GueX, J. 1991: BioGraph: un nouveau programme de construction des corrélations biochronologiques basées sur les associations unitaires. Bulletin de la Société Vaudoise des Sciences Naturelles, 80 (3), $317-340$.
SCHAAF, A. 1985: Un nouveau canevas biochronologique du Crétacé inférieur et moyen: les biozones à radiolaires. Sci. géol. (Strasbourg) Bull., 38(3), 227-269.

Souinabol, S. 1903: Radiolarie fossile di Teolo (Euganei). Atti e memorie del'Academia di scienze, lettere ed arti. Padova, new series, 19, 127-130.

Souinabol, S. 1904: Radiolarie cretacee degli Euganei. Atti e memorie del'Academia di scienze, lettere ed arti. Padova, new series, 20, 171-244.

Squinabol, S. 1914: Contributo alla conoscenza dei Radiolarii fossili del Veneto. Appendice - Di un genera di Radiolari caratteristico del Secondario (Contribution to the knowledge of fossil Radiolaria. Appendix - On a genus of Radiolaria characteristic of the Mesozoic). Memorie dell'Istituto geologico della R. Universita di Padova, 2, 249-306.

TAKETANI, Y. 1982: Cretaceous radiolarian biostratigraphy of the Urakawa and Obira areas, Hokkaido. Sci. Rep. Tohoku Univ. Series 2: Geology = Tohoku Daigaku Rika Hokoku. Dai 2: Shu Chishitsugaku, 52(1-2), 1-76.

Thiébault, F., De wever, P., Fleury, J.J. \& Bassoulet, J.P. 1981: Précisions sur la série stratigraphique de la nappe du Pinde-Olonos de la presqu'île de Koroni (Péloponnèse méridional - Grèce): l'âge des Radiolarites - (Dogger - Crétacé supérieur). Ann. Soc. Géol. Nord, 100, 91-105.

Thurow, J. 1988: Cretaceous radiolarians of the North Atlantic Ocean; ODP Leg 103 (sites 638, 640, and 641) and DSDP legs 93 (Site 603) and 47B (Site 398). In: Boillot, G., Winterer, E.L., (Eds.): Proceedings of the Ocean Drilling Program, Scientific results (Galicia margin; covering Leg 103 of the cruises of the drilling vessel JOIDES Resolution, Ponta Delgada, Azores, to Bremerhaven, Germany, 25 April 1985-19 June 1985), 103, 379-418. Texas A. \& M. University, Ocean Drilling Program, College Station, TX, United States.

Vernez, G. 1990: Etude géologique et minéralogique de la Vallée de Paliominos entre Karnezeika et Stavropodhi. Unpublished diploma thesis, University of Lausanne.

VishneVSKAYA, V. S. 2001: Jurassic to Cretaceous radiolarian biostratigraphy of Russia. GEOS, Moscow, 376 p.

VRIELYNCK, B. 1981: Evolution paléogéographique et structurale de la presqu'île d'Argolide (Grèce). Revue de géologie dynamique et de géographie physique, 23(4), 277-288.

Manuscript received January 2004

Revision accepted February 2005

Plate 1

SEM-illustrations of Upper Cretaceous radiolarians from Karnezeika, Argolis Peninsula (Greece)

Figures 1-2 Acaeniotyle rebellis O’DOGHERTY 1994 Al68_06 (Figs. 1 and 2)

Figure 3 Acaeniotyle sp. A Al74_300

Figure $4 \quad$ Acaeniotyle sp. B Al70_090

Figures 5-6 Archaeocenosphaera (?) mellifera O'DOGHERTY 1994 Al72_190 (Figs. 5 and 6)

Figures 7-8 Archaeocenosphaera (?) sp. Al73_320 (Figs. 7 and 8)

Figures 9-10 Triactoma cellulosa FOREMAN 1973 Al74_300 (Figs. 9 and 10)

Figures 11-12 Triactoma hexeris O'DOGHERTY 1994 Al73_320 (Figs. 11 and 12)

Figure $13 \quad$ Triactoma sp. aff. T. hexeris O'DOGHERTY 1994 Al72_190

Figure 14 Pseudoacanthosphaera galeata O'DOGHERTY 1994 Al73_320

Figure 15 Pseudoacanthosphaera superba (SQUINABOL 1904) Al74_300

Figure $16 \quad$ Pseudoacanthosphaera sp. aff. P. spinosissima (SOUINABOL 1904) Al73_320

Figure $17 \quad$ Pseudoacanthosphaera (?) sp. Al73_320

Figure $18 \quad$ Tetracanthellipsis euganeus SQUINABOL 1903 Al73_320

Figures 19-20 Praeconocaryomma universa Pessagno 1976 Al72_190 (Figs. 19 and 20)

Figures 21-22 Praeconocaryomma californiaensis PESSAGNO 1976 Al72_190 (Figs. 21 and 22)

Figures 23-24 Praeconocaryomma lipmanae Pessagno 1976 Al74_300 (Figs. 23 and 24)

Figures 25-26 Praeconocaryomma sp. Al74_300 (Fig.25 and 26)

Figures 27-28 Crucella messinae Pessagno 1971 Al68_060 (Fig. 27), Al72_190 (Fig. 28)

Figures 29-30 Crucella cachensis PESSAGNO 1971 Al74_300 (Fig. 29), Al73_320 (Fig. 30)

Figure 31 Halesium triacanthum (SQUINABOL 1903) sensu O'DOGHERTY 1994 Al68_060

Figures 32-33 Halesium sp. Al68_060 (Fig. 32), Al73_320 (Fig. 33)

Figures $34 \quad$ Pessagnobrachia sp. Al74_300 


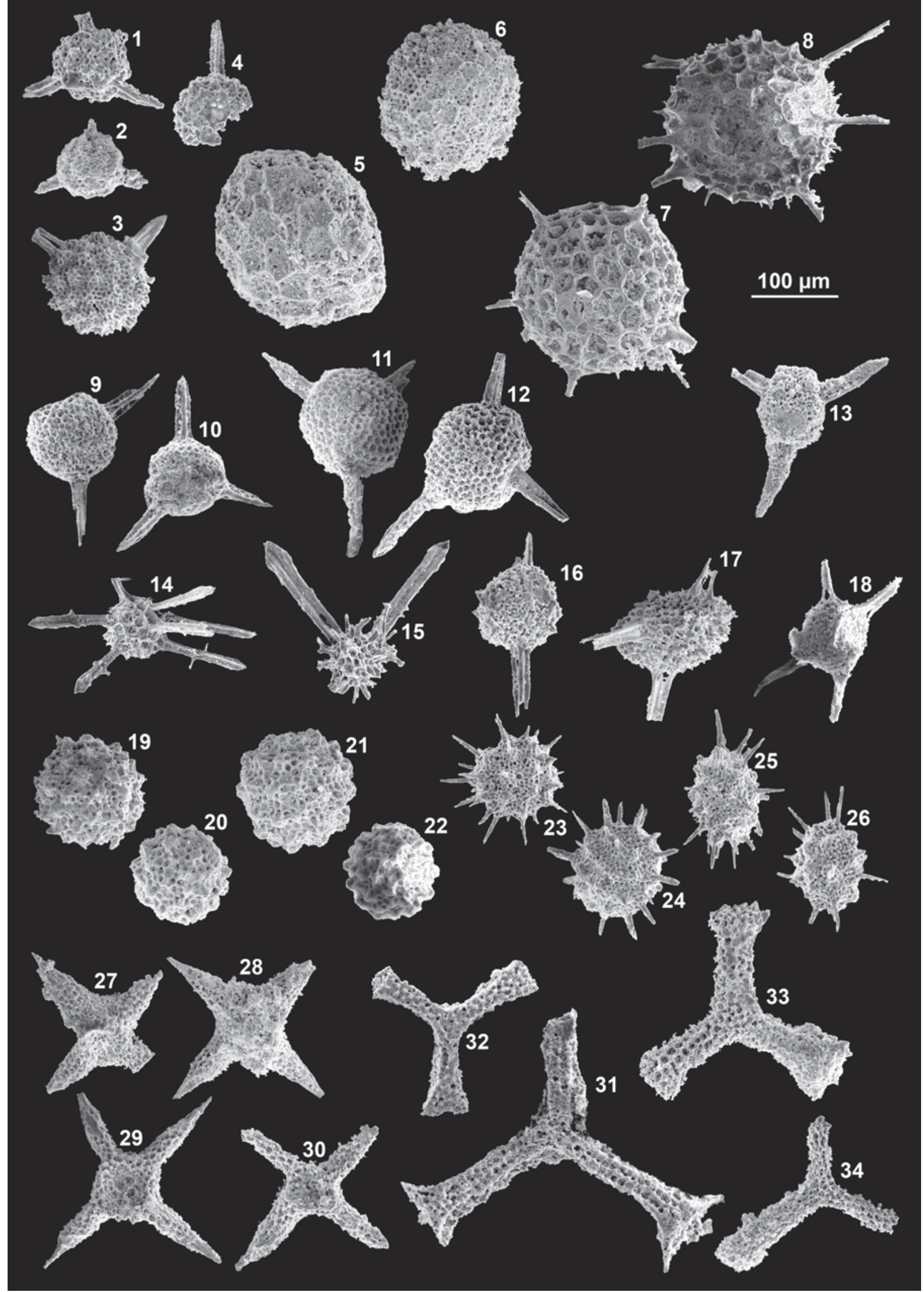


Plate 2

SEM-illustrations of Upper Cretaceous radiolarians from Karnezeika, Argolis Peninsula (Greece)

Figures 1-2 Dactyliodiscus sp. Al72_190 (Figs. 1 and 2)

Figures 3-4 Pseudoaulophacus sculptus (SQUINABOL 1904) sensu O’DOGHERTY 1994 Al72_190 (Figs. 3 and 4)

Figures 5-6 Pseudoaulophacus putahensis PESSAGNo 1972 Al73_320 (Figs. 5 and 6)

Figures 7-8 Patellula helios (SQUinABOL 1903) sensu O'DOGHERTY 1994 Al74_300 (Figs. 7 and 8)

Figures 9-10 Patellula ecliptica O’DOGHERTY 1994 Al73_320 (Figs. 9 and 10)

Figures 11-12 Patellula heroica O'DOGHERTY 1994 Al74_300 (Figs. 11 and 12)

Figure $13 \quad$ Patellula sp. Al72_190

Figures 14-15 Acanthocircus venetus (SQuinabol 1914) sensu O'DOGHERTy 1994 Al72_190 (Fig. 14), Al73_320 (Fig. 15)

Figures 16-17 Acanthocircus tympanum O'DOGHERTY 1994 Al74_300 (Fig. 16), Al73_320 (Fig. 17)

Figures 18-19 Acanthocircus hueyi (PESSAGNo 1976) sensu O’Dogherty 1994 Al74_300 (Fig. 18), Al73_320 (Fig. 19)

Figures 20-21 Dictyomitra formosa Souinabol 1904 Al72_190 (Fig. 20), Al73_320 (Fig. 21)

Figure $22 \quad$ Dictyomitra sp. cf. D. formosa Souinabol 1904 Al72_190

Figures 23-24 Dictyomitra montisserei (SQuinabOL 1903) sensu O'DOGHeRTy 1994 Al68_060 (Fig. 23), Al72_190 (Fig. 24)

Figure 25 Dictyomitra urakawensis TAKETANI 1982 Al72_190

Figure $26 \quad$ Torculum coronatum (SQUINABOL 1904) Al72_190

Figures 27-28 Pseudodictyomitra pseudomacrocephala (Souinabol 1903) Al73_320 (Figs. 27 and 28)

Figures 29-30 Stichomitra communis SQuinabOL 1903 Al73_320 (Figs. 29 and 30)

Figures 31-32 Stichomitra stocki (CAMPBEll \& Clark 1944) sensu O'DOGHerty 1994 Al68_060 (Fig. 31), Al73_320 (Fig. 32)

Figure 33

Afens liriodes RIEDEL \& SANFILIPPO 1974 Al74_300 

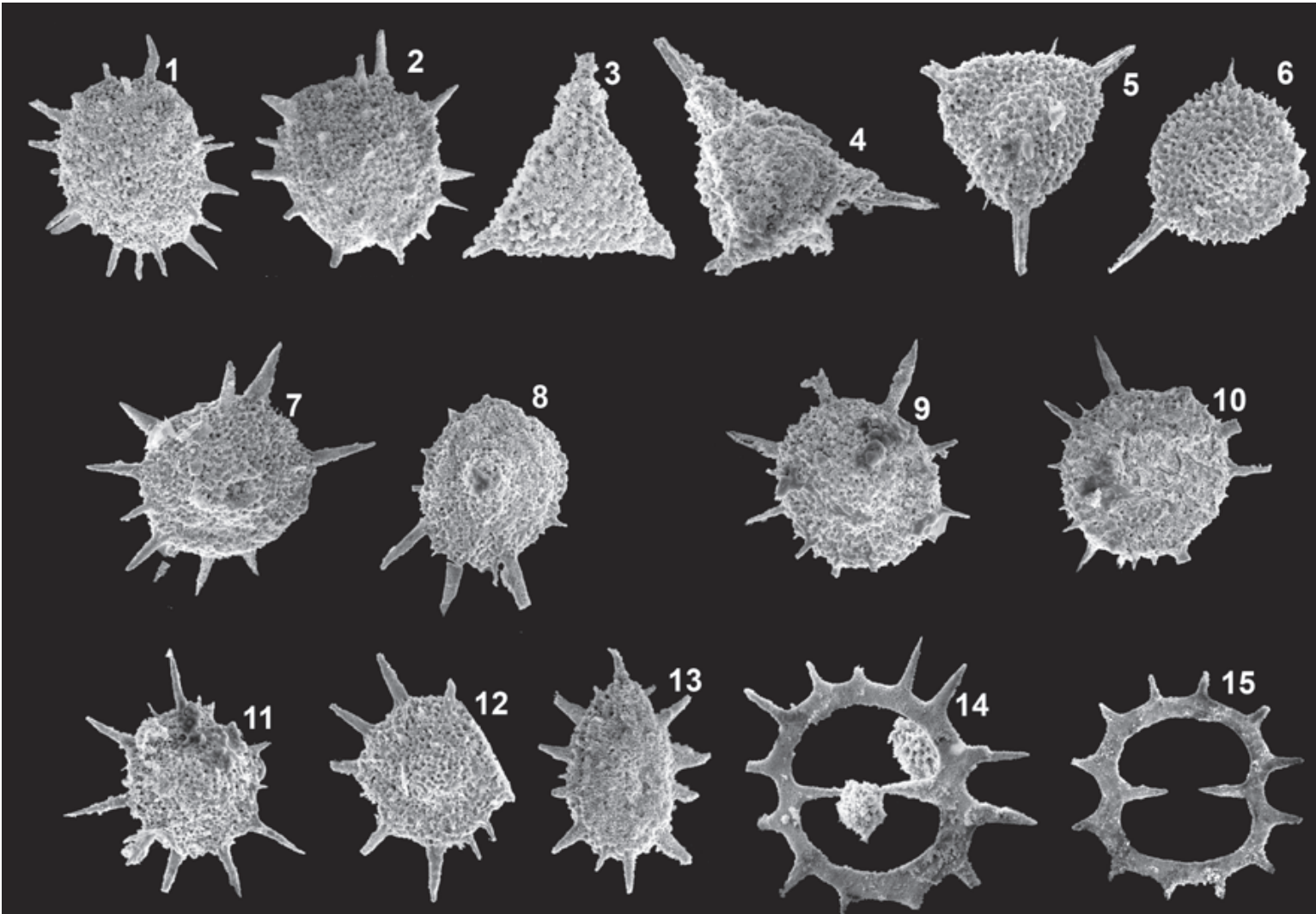

\section{$100 \mu \mathrm{m}$}
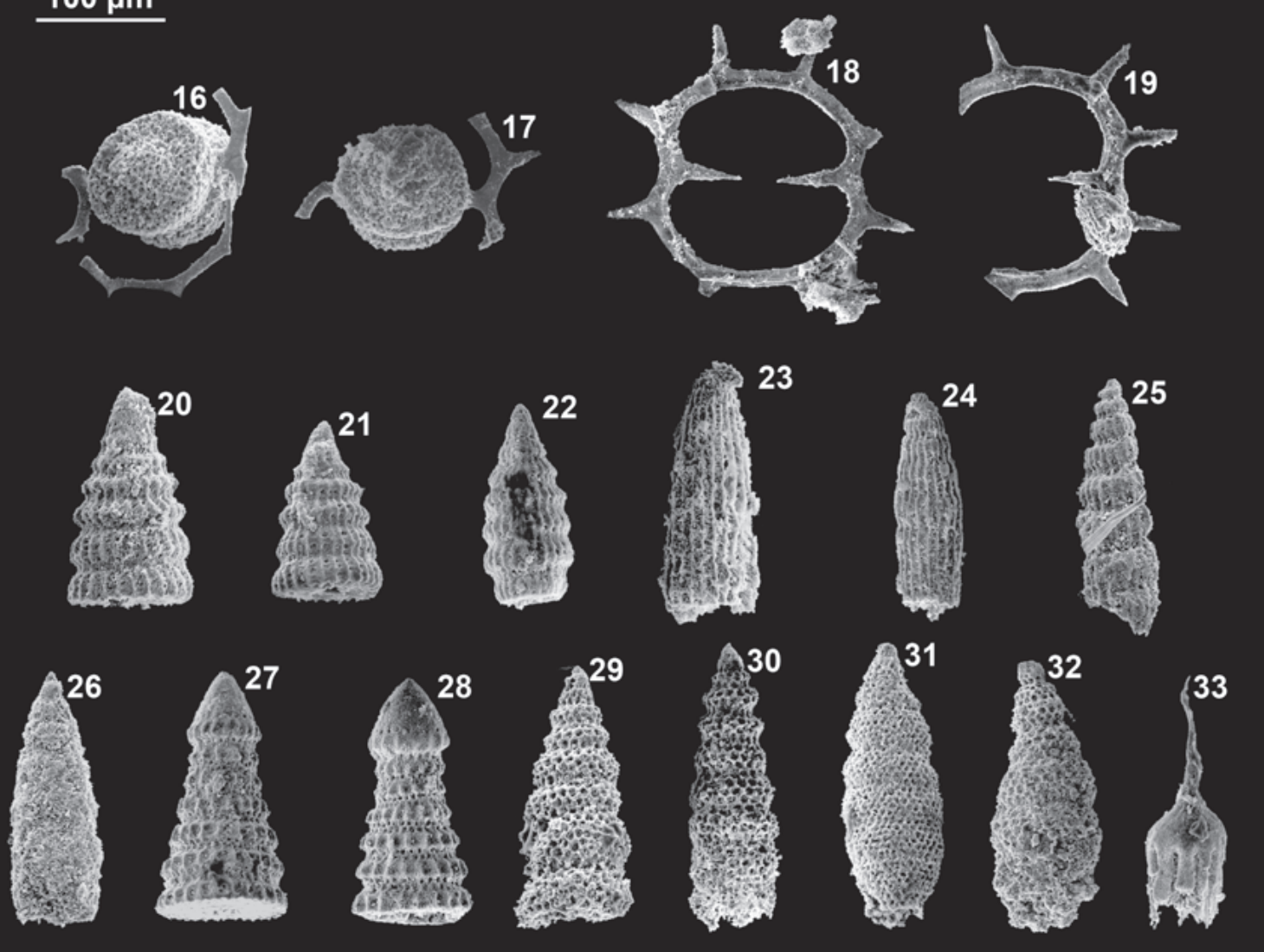

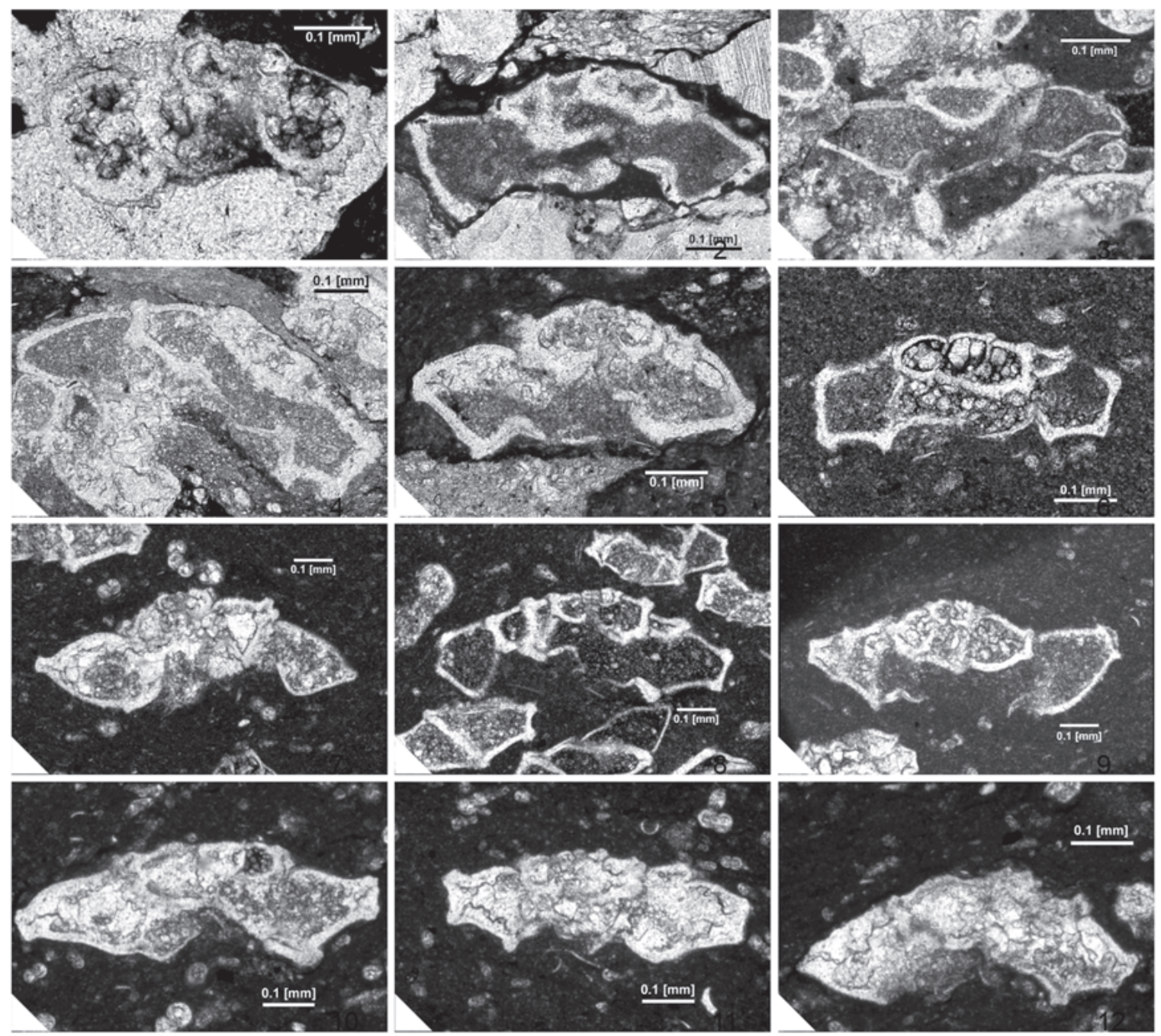

Plate 3

Optical microscope illustrations of Upper Cretaceous planktonic foraminfers from Karnezeika, Argolis Peninsula (Greece)

Figure 1 Helvetoglobotruncana helvetica (BOLLI 1945) CKAR2

Figure 2 Marginotruncana marianosi (DougLAS 1969) CKAR7

Figure 3 Marginotruncana schneegansi SIGAL 1952 CKAR13

Figure 4 Marginotruncana coronata (BOLLI 1945) CKAR13

Figure 5 Marginotruncana sigali (REICHEL 1950) CKAR13

Figure 6 Marginotruncana pseudolinneiana PESSAGNO 1967 CKAR14

Figure 7 Marginotruncana schneegansi SIGAL 1952 CKAR14

Figure 8 Marginotruncana sinuosa PORTHAULT 1970 CKAR14

Figure 9 Dicarinella primitiva (DALBIEZ 1955) CKAR14

Figure 10 Marginotruncana pseudolinneiana PESSAGNO 1967 Al76_1350

Figure 11 Marginotruncana pseudolinneiana PESSAGNO 1967 Al76_1350

Figure 12 Marginotruncana sigali (REICHEL 1950) Al76_1350 